\title{
Using a distance map and an active contour model to segment the carotid artery boundary from the lumen contour in proton density weighted magnetic resonance images
}

\author{
Danilo Samuel Jodas ${ }^{\mathrm{a}, \mathrm{e}}$, Maria Francisca Monteiro da Costa $^{\mathrm{b}}$, Tiago A. A. Parreira ${ }^{\mathrm{c}}$, Aledir Silveira \\ Pereira $^{\mathrm{d}}$, João Manuel R. S. Tavares ${ }^{\mathrm{e}, *}$ \\ CAPES Foundation, Ministry of Education of Brazil ${ }^{a}$, IFE Neurorradiologia, Serviço de Neurorradiologia, Centro Hospitalar \\ São João ${ }^{b}$, AH Neurorradiologia, Serviço de Neurorradiologia, Centro Hospitalar São João ${ }^{c}$, Universidade Estadual Paulista \\ "Júlio de Mesquita Filho" ", Instituto de Ciência e Inovação em Engenharia Mecânica e Engenharia Industrial, Faculdade de \\ Engenharia, Universidade do Porto ${ }^{e}$ \\ ${ }^{a}$ Brasília - DF, 70040-020, Brazil \\ ${ }^{b}$ Alameda Professor Hernâni Monteiro, 4200-319 Porto, Portugal \\ ${ }^{c}$ Alameda Professor Hernâni Monteiro, 4200-319 Porto, Portugal \\ ${ }^{d}$ Rua Cristóvão Colombo, 2265, 15054-000, S. J. do Rio Preto, Brazil \\ ${ }^{e}$ Rua Dr. Roberto Frias, s/n, 4200-465, Porto, Portugal
}

\begin{abstract}
Segmentation methods have assumed an important role in image-based diagnosis of several cardiovascular diseases. Particularly, the segmentation of the boundary of the carotid artery is demanded in the detection and characterization of atherosclerosis and assessment of the disease progression. In this article, a fully automatic approach for the segmentation of the carotid artery boundary in Proton Density Weighted Magnetic Resonance Images is presented. The approach relies on the expansion of the lumen contour based on a distance map built using the gray-weighted distance relative to the centre of the identified lumen region in the image under analysis. Then, a Snake model with a modified weighted external energy based on the combination of a balloon force along with a Gradient Vector Flow-based external energy is applied to the expanded contour towards the correct boundary of the carotid artery. The average values of the Dice coefficient, Polyline distance, mean contour distance and centroid distance found in the segmentation of 139 carotid arteries were $0.83 \pm 0.11,2.70 \pm 1.69$ pixels, $2.79 \pm 1.89$ pixels and $3.44 \pm 2.82$ pixels, respectively. The segmentation results of the proposed approach were also compared against the ones obtained by related approaches found in the literature, which confirmed the outstanding performance of the new approach. Additionally, the proposed weighted external energy for the Snake model was shown to be also robust to carotid arteries with large thickness and weak boundary image edges.
\end{abstract}

Keywords: Medical imaging, Magnetic resonance imaging, Image segmentation, Snake model,

Gray-weighted distance

\footnotetext{
${ }^{*}$ Corresponding author. Tel.: +35122508 1487; fax: +35122508 1445

Email addresses: danilojodas@gmail.com (Danilo Samuel Jodas), mariacostapd@gmail.com (Maria Francisca Monteiro da Costa), tiagoparreira@gmail.com (Tiago A. A. Parreira), aledir@sjrp.unesp.br (Aledir Silveira Pereira), tavares@fe.up.pt (João Manuel R. S. Tavares)

URL: http://www.fe.up.pt/ tavares (João Manuel R. S. Tavares)
} 


\section{Introduction}

Cardiovascular diseases are a major cause of death and disability in the world. The most usual underlying disease process is atherosclerosis, which is still the most dangerous disease that affects the majority of people around the world [1]. The accumulation of fatty material and cholesterol in the walls of the arterial system

5 is the underlying condition to the formation of the so-called atherosclerotic plaques, which can progressively obstruct the blood through the artery and lead to heart attacks and strokes. Therefore, an early diagnosis is important to rapidly establish the correct treatment planning for preventing the onset of such symptoms.

Magnetic resonance imaging (MRI) has been widely used in non-invasive image-based diagnosis of the carotid artery. Particularly, magnetic resonance (MR) images have been successfully used in the characterization of atherosclerotic plaques, enabling the assessment of the disease progression [2]. Computer-aided methods for the analysis of the carotid artery in MR images play an important role to expedite the identification and assessment of possible atherosclerosis and, consequently, the design of the best treatment plan to prevent future symptomatic events. [3, 4, 4 ].

Despite the importance of the lumen contour for predicting the possible blood flow obstruction caused by atherosclerotic plaques, carotid wall boundaries are essentially worth in assessing the morphology of the arteries considering the use of metrics like the wall thickness, for example, and even necessary as the first step towards their identification in images with other surrounding structures. However, the manual delineation of the lumen and carotid wall boundaries is a very arduous and time-consuming task; particularly, when taking into account the high number of slices acquired in a single imaging exam. Approaches based on surface graph cuts 6, 7], ellipse fitting [8, 9], difference of Gaussian 10] and active contour models 11, 12, 13, 14, 15, 16, 17, 18, 19, 20] have been proposed for the segmentation of the lumen and carotid wall boundaries in MR images. However, the main limitation of these methods is the necessity of manual interventions and the expansion of the lumen contour according to a pre-defined value as in Ladak et al. [12], van 't Klooster et al. 15], Saba et al. 17] and Gao et al. [18].

Besides the approaches based on active contour models, the segmentation of structures in medical images can also be formulated as level set curves in order to tackle possible topological changes in the structures of interest and the similarity of its grayscale intensities with the ones of neighbour structures [21, 22, 23]. Regarding the latter challenge, Wang et al. 24] proposed a shape-intensity level set method to segment the boundary of the liver in Computed Tomography (CT) images of the abdominal region. Despite the reported effectiveness, level set methods may still suffer to properly adapt the segmentation contours in regions of inhomogeneous grayscale intensity, a common aspect of the images herein tackled. On the other hand, active contour models are still worth to handle tasks where the purpose is the identification of a single boundary.

Heuristic values can fail to properly expand the lumen contour outward the correct lumen region. Furthermore, the gradient information usually employed in active contour models is also inadequate to successfully tackle weak boundaries that are commonly found on MR images of the carotid artery. Regarding the former challenge, the expansion of the lumen contour should not depend on any heuristic value in order the segmen- 
tation of the lumen and carotid wall boundaries can be fully automatic and robust, which is highly demanded to rapidly provide to the clinical experts the region of the vessel to be evaluated. The latter difficulty regards the adoption of suitable image processing techniques to extract useful information about regions of interest that have weak boundaries, but have an important role in the achievement of the best segmentation results. These two difficulties were the main concerns of this study, which leads us to propose a novel approach for adressing at the same time the fully automatic segmentation of carotid arteries and the weak boundaries in regions of interest that occasionally lead the contours to incorrect locations.

Therefore, this article proposes a novel fully automatic approach to segment the lumen and outer boundaries of the carotid artery in Proton Density Weighted (PDW) MR images. The proposed approach performs the segmentation of the carotid artery boundary using a gray-weighted distance map and a Snake model with a modified external energy that relies on Gradient Vector Flow and balloon forces. Briefly, the first step of the new approach concerns the identification of the lumen contour using the mean roundness criterium proposed in Jodas et al. [25]. Then, since the magnitude of the gradient along the lumen region prevents the lumen contour to reach the boundary of the carotid artery, the gray-weighted distance map is employed to expand the contour outward the lumen region towards the carotid artery boundary. Then, the region inside the expanded contour is discarded and the contour is used to initiate a Snake model in order to segment the carotid artery boundary. Since the Snake model is a particular case of deformable models, the previously identified lumen contour may be further improved using the concept of curve evolution to find the boundary of the carotid artery based on geometrical properties of the contour and image-based information.

This study provides three main contributions: 1) The use of the gray-weighted distance map to prevent the lumen contour to be trapped inside the lumen region by the high magnitude of the gradient surround the lumen contour. Consequently, the proposed approach assists the used active contour model to automatically expand the lumen contour outward the lumen region; 2) the combination of a balloon force along with a Gradient Vector Flow-based external energy in the Snake model to also handle the segmentation of the boundaries of carotid arteries of large thickness and weak image edges. The use of the intensities' entropy and the analysis of the gradient vectors ahead the points of the contour to be deformed are used to improve the segmentation of the carotid wall boundary; 3) the detailed assessment of the accuracy of the proposed approach using a challenging PDW MR image dataset.

The remainder of this article is organized as follow: Section 2 describes the proposed automatic segmentation approach. Section 3 presents segmentation results obtained by the proposed approach and their comparison against the corresponding manual delineations. The advantages and limitations of the proposed approach are identified in Section 4 Finally, the conclusions are drawn in Section 5 


\section{Materials and Methods}

This investigation was performed using MR images of the carotid artery that were beforehand used in the research carried out by van Engelen et al. [26] and kindly provided by the authors on request. The used images were collected according to the Medical Committee of the Erasmus Medical Center, and all assessed patients provided their written consensus for the use of the acquired images. Additional information about the MRI scans and related ethical clearance is available in van Engelen et al. [26]. All the images included in the used dataset represent cropped regions that contain carotid arteries acquired by multiple imaging modalities. The MR images having a perfect registration, which was performed using the approach suggested in van Engelen et al. [26], with the corresponding histology images were cropped to obtain the final regions of interest of the carotid arteries [26]. Five MRI scans obtained from thirteen patients: T1-weighted (T1W), Proton Density Weighted (PDW), Time-of-Flight (TOF), and two 3D-T1W scans, are contained in the dataset. Each included MRI scan is formed of nearly $17.7 \pm 4.8$ slices per patient, having each one a pixel size of $0.25 \mathrm{~mm} \times 0.25 \mathrm{~mm}$.

In order to overcome the lack of some manual delineations in the original dataset and some misalignments of the provided contours due the registration process described in [26], which could affect the quantitative analysis of the segmentation accuracy, the lumen and carotid walls were manually delineated on the 230 PDW MR images of the thirteen patients. The delineations were made by a physician of the Neuroradiology Department from the Centro Hospitalar São João, in Porto, Portugal, with the supervision of an experienced physician from the same department. The manual contours of the lumen and carotid walls of the images belonging to eight patients were established based on the PDW MR images only; the delineations of the images belonging to four patients were made with the additional visual examination of the corresponding 3D-T1W MR images; and the PDW MR images of one patient were not manually segmented due to their low quality and the presence of strong artefacts; additionally, the images of two patients were not used due to the impossibility of identifying the structures of interest. Hence, 185 PDW MR images with manual delineations of the lumen and carotid boundaries were used in this study.

\subsection{Proposed approach}

The diagram of the proposed automatic approach for segmenting the carotid wall boundary in PDW MR images is depicted in Figure 1

Here, the segmentation of the lumen boundary was performed using a method already developed and proposed in our previous study [25], which relies on a circularity index calculated from each region identified no by the K-means algorithm with subtractive clustering. The method described in [25] refers to an already developed approach for the segmentation of the lumen region in images of magnetic resonance and intravascular ultrasound imaging techniques. The identified lumen contour is then submitted to the vessel wall segmentation stage to expand it to the outer boundary of the carotid artery. In the vessel wall segmentation 


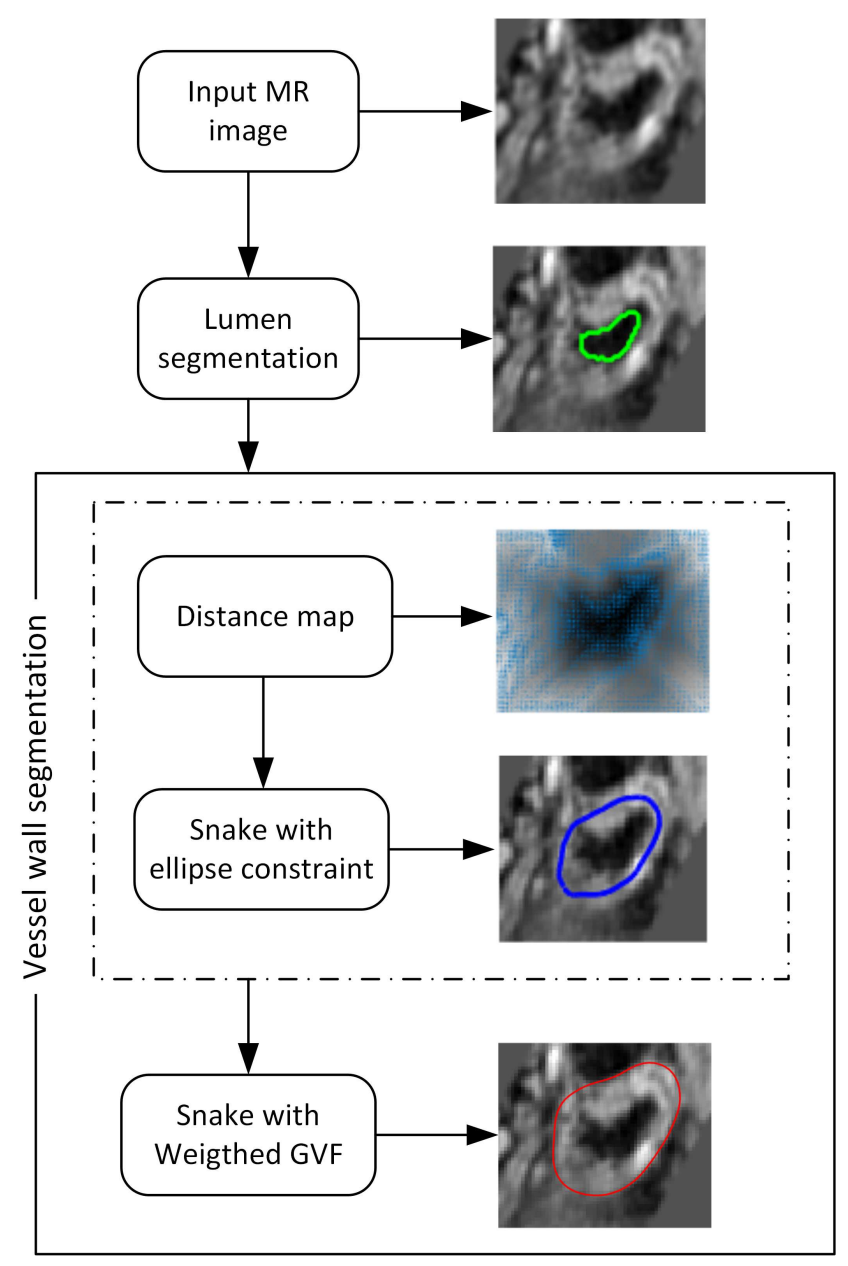

Figure 1: Proposed approach for segmenting the carotid artery boundary in PDW MR images.

stage, the lumen contour is expanded according to two steps: the first step consists in expanding the contour outward the lumen region, by means of the application of a Snake active contour with ellipse constraint in the grayscale distance map of the input MR image; and the second step completes the expansion to the true boundary of the carotid artery, based on a Snake active contour with a suitable weighted external energy.

\subsubsection{Distance map}

Edge detection operators rely on the gradient of the input image to find significant variations on the grayscale intensities of the existent regions. Usually, in Black-Blood MR images of the carotid artery, the lumen is a region with low intensity values surrounded by high intensity values corresponding to the region of the vessel wall. Hence, significant variations of the grayscale intensity between the lumen and vessel wall regions represent a challenge to expand the lumen contour towards the boundary of the carotid artery of interest.

Usually, the external energies used in the Snake model rely on the magnitude of the gradient vectors to decrease the evolution of the initial contours towards the boundaries of the structures of interest. However, 
the high magnitude of the gradient vectors around the lumen region can hinder the expansion of the identified lumen contour towards the boundary of the carotid artery under analysis. Approaches based on heuristic values used in order to expand the lumen contour in the direction of the boundary of the carotid artery, may efficient and dynamic approach to avoid the edges between the lumen and carotid wall is proposed in this study.

The distance transform is a technique applied on binary or grayscale images to assign a value to each pixel of the structure of interest that represents the nearest distance to the pixels of the image background. and column 2 of Figure 2 (a) is equal to $\frac{(13+6)}{2}=9.5$, which is the value in line 3 and column 2 illustrated in Figure 2(b). The same principle is followed to calculate the distances for all the neighbor values from centre value 13. The arrows in Figure 2(b) indicate the paths that follow the minimum distances from the centre position to the borders of the depicted matrix. For example, the distance from centre value 13 and value 4 located in line 3 and column 1 in Figure 2(a) is calculated as $\min \left(9.5+\frac{(6+4)}{2}, 9+\frac{(5+4)}{2}=\min (14.5,13.5)\right.$, which gives values $13,5,4$ as the minimum path from the centre value to the border located in line 3 and column 1 of the example depicted in Figure 2

where $f\left(p_{i}-1\right)$ and $f\left(p_{i}\right)$ are the intensity of two adjacent pixels along the minimum cost path $t_{f}(P)$, and $l$ is the length of the path $P$. Starting from pixel $p$, the minimum cost relatively to pixel $q$ is calculated taking into account the $3 \times 3$ neighbors of each pixel along the path according to Equation 1 . The geodesic time assures that there is only one path with minimum cost between pixels $p$ and $q$. Hence, the geodesic time is the smallest amount of time, i.e., cost, between pixels $p$ and $q$ :

$$
t_{f}(p, q)=\min \left\{t_{f}(P) \mid P \text { connects } p \text { and } q\right\} \text {. }
$$

Figure 2 illustrates an example of the gray-weighted distance calculated from a centre pixel to each pixel at the border of a $5 \times 5$ matrix.

For each value of the matrix depicted in Figure 2(a), Equation 1 is applied to calculate the distance between current value $f\left(p_{i}\right)$ and previous value $f\left(p_{i}-1\right)$. For example, the distance between the path represented by centre value $13\left(f\left(p_{i}-1\right)\right)$ framed in red in Figure 2(a) and value $6\left(f\left(p_{i}\right)\right)$ located in line 3 


$\begin{array}{rrrrr}17 & 24 & 1 & 8 & 15 \\ 23 & 5 & 7 & 14 & 16 \\ 4 & 6 & 13 & 20 & 22 \\ 10 & 12 & 19 & 21 & 3 \\ 11 & 18 & 25 & 2 & 9\end{array}$

(a)

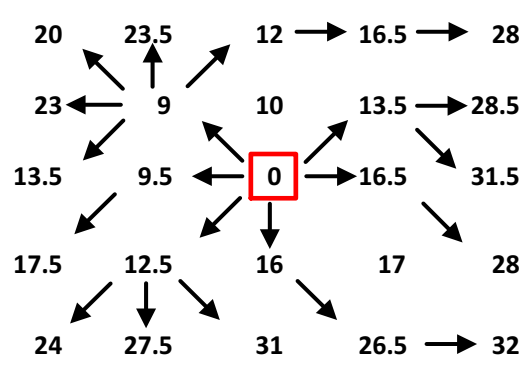

(b)

Figure 2: Example of application of the gray-weighted distance on a $5 \times 5$ matrix: a) The $5 \times 5$ matrix with grayscale intensities; b) The gray-weighted distance calculated from the pixel framed in red to each pixel at the border of the matrix. (The arrows indicate the path with minimum cost.)

As shown in Figure 2, the distance along the path connecting the centre pixel to each pixel at the border of the matrix increases according to the intensity values. The higher the pixel intensities along the path, the larger will be the distance from the starting pixel to the ending pixel. Considering the common low intensity of the lumen region of the carotid artery in Black-Blood MR images, the distances from the centre of the lumen to all pixels at the border of the input image start to increase significantly as the paths reach the edge between the lumen and the vessel wall regions. Hence, the expansion of the identified lumen contour beyond the boundary of the lumen region can be performed by decreasing the contour evolution as the values of the distance map increase. Examples of the gray-weighted distance applied to PDW MR images of carotid arteries are depicted in Figure 3
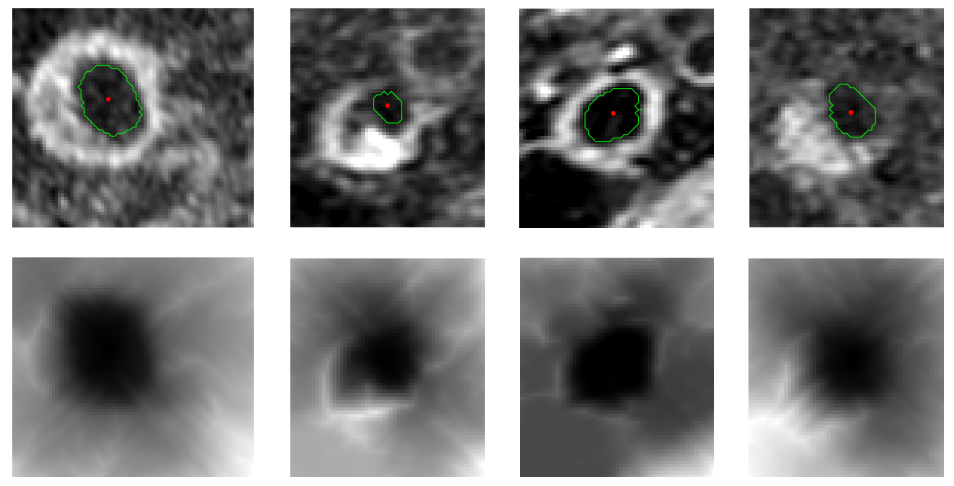

Figure 3: Examples of the gray-weighted distance applied to PDW MR images of carotid arteries: The images on the top row represent the ones with the segmented lumen contours in green (the starting points of the gray-weighted distance are the red dots that represent the centres of the lumen contours). The images on the bottom row show the corresponding distance maps calculated by Equation 1

As shown in Figure 3, the grayscale intensities of the images corresponding to the distance maps (on the bottom row) represent the distances from the centres of the lumen contours to all pixels at the border of the images. The higher the intensity values are, the larger will be the distances from the centre of the lumen. 
The distances inside the lumen region start to increase close to the edges of the lumen boundary.

\subsubsection{Active contour model}

Deformable models have been widely used in the segmentation of boundaries of structures presented in medical images [31, 32, 33, 34, 35]. Active contours, also known as Snakes, were proposed by Kass et al. 36] to find boundaries of regions in images through the evolution of curves, i.e., contours, controlled by internal and external energies. The active contour model proposed by Kass et al. [36] has been extensively used in tasks of image processing and analysis, particularly in the segmentation of medical images. However, the gradient of the input image commonly used as external energy provides limited capture range and poor convergence to concavities. Hence, the initial contour needs to be placed close to the boundary of the structure of interest. Therefore, balloon forces [37] were proposed to increase the amplitude and range of the contour to be evolved:

$$
F_{\text {balloon }}=k n(s)
$$

where $n(s)$ is a unit vector perpendicular to each point of the contour to be deformed, and $k$ is a weight that defines the amplitude of the unit vector force. The sign of $k$ defines the orientation of the contour, i.e., the ability to inflate or deflate it around the structure of interest. A positive value of $k$ will make the contour to inflate until it fits the boundary of interest, whereas a negative value will be responsible to deflate the contour. Balloon forces have been successfully applied to increase the pressure force of active contour models, leading the contours to fit the boundaries of interest even when the initial contours are distant from the structures of interest. However, balloon forces may cause the segmentation contour to leak weak image edges due to the amplitude and strengthen of the unit vectors.

$\mathrm{Xu}$ and Prince [38] proposed a new external force called Gradient Vector Flow (GVF) to increase the capture range and tackle the concavities problem by diffusing the gradient vectors of an edge map calculated from the input image. Although successfully used as an external energy for active contour models, the main disadvantage of the GVF method is the inability to deal with weak image edges that can cause the contour to leak the true boundary. Liu and Bovik [39] proposed a new external energy for the Snake model based on the decomposition of the Laplacian operator. This new external energy is called Neighbourhood-Extending and Noise-Smoothing Gradient Vector Flow (NNGVF), which is based on the convolution of two templates used to maintain the low-frequency components of the image obtained by a gradient operator, suppress noisy artefacts and increase edge-preservation. Since the convolution is performed in larger neighbourhoods, the NNGVF performs noise reduction and captures more information to calculate the diffusion vectors in regions with weak edges.

Although the NNGVF provides better results when compared to the GVF, the evolution of the contour in structures with large areas is still the most challenge in the segmentation of medical images. Examples of segmentation results obtained by the Snake model with the NNGVF applied to PDW MR images of carotid arteries are shown in Figure 4. 

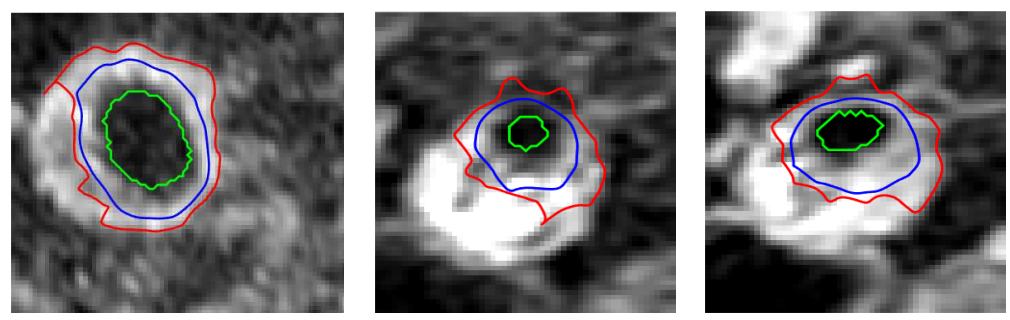

Figure 4: Examples of incomplete convergence of the lumen contours to the boundaries of carotid arteries resulting from the Snake model with the NNGVF applied to PDW MR images. (The identified lumen contours are in green, the blue contours are the lumen contours expanded beyond the boundaries of the lumen regions by using the images resulting from the gray-weighted distance, and the red contours are the results of the Snake model with the NNGVF initialized with the blue contours.)

In Figure 4 the lumen contours identified by the method proposed in [25] are represented in green; the blue contours are the lumen contours expanded beyond the boundaries of the lumen regions by using the Snake model initialized with the green contours in the images resulting from the gray-weighted distance; the contours in red are the results of the Snake model with the NNGVF initialized with the blue contours. Here, the incomplete convergence of the initial contours in blue to the true boundaries of the carotid arteries is due to the variations of the grayscale intensities inside the carotid wall and the high distance to the boundaries of interest.

In order to overcome the usual limited range covered by the GVF, Khadidos et al. [40] proposed a new external energy for the Snake model based on the combination of the GVF and balloon-based forces. Hence, a weighted factor based on the intensity of the magnitude of the gradient and the difference between the directions of the balloon forces, and the gradient vectors was introduced to control the evolution of the initial contour [40]:

$$
E_{\text {ext }}=\left(F_{\text {balloon }} *(1-\Omega)\right)+\left(F_{G V F} * \Omega\right),
$$
calculated from the GVF based force and $\Omega$ is defined as:

$$
\Omega=h^{1-(A D-\epsilon)} .
$$

Parameter $\Omega$ represents a value between 0 (zero) and 1 (one) measured based on the intensities of the pixels ahead each point of the contour. Therefore, $\Omega$ is a weighted factor that determines which gradient forces (GVF, balloon force or a combination of both) need to be applied at each point of the contour. Constant ${ }_{210} \epsilon=0.001$ is employed to avoid power by zero in cases where $A D=1$. The value of $h$ at each pixel $(x, y)$ of the contour to be deformed is defined as the average value of the intensities inside a semi-circular region centred at $(x, y)$ :

$$
h(x, y)=\frac{1}{N} \sum_{(i, j) \in S} f(i, j)
$$


where $f(i, j)$ is the intensity value at pixel $(i, j)$ of the image with edges detected by a gradient operator, $S$ is the region inside the semi-circle centred at contour pixel $(x, y)$, and $N$ is the number of pixels of region $S$.

The value of $A D$ at each contour pixel $(x, y)$ is defined as the average of the difference between the angle of the balloon force and the angle of each gradient vector inside a cone-shaped region ahead contour pixel $(x, y)$ :

$$
A D(x, y)=\frac{1}{M * \pi} \sum_{(i, j) \in T} \theta(i, j),
$$

where $\theta(i, j)$ is the angle between the balloon force at contour pixel $(x, y)$ and the gradient vector at pixel $(i, j), T$ contains the pixels ahead contour pixel $(x, y)$ that are inside the cone-shaped region, and $M$ is the number of pixels of this cone-shaped region. The semi-circular and cone-shaped regions are depicted in Figure

5.

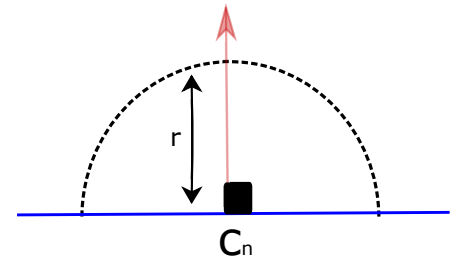

(a)

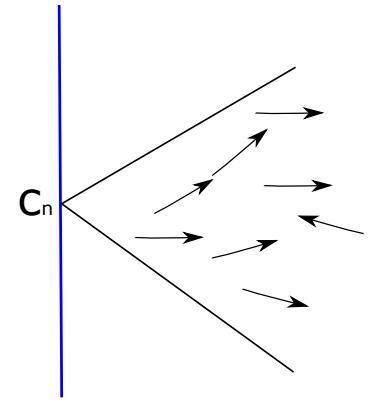

(b)

Figure 5: Representation of the semi-circular region $S$ in (a) and the cone-shaped region $T$ in (b), both positioned perpendicularly to contour pixel $\mathrm{C}_{n}$. The blue line represents the contour to be deformed; parameter $r$ in (a) represents the radius of the semicircular region; and the black arrows illustrated in (b) refers to the gradient vectors ahead contour pixel $\mathrm{C}_{n}$. (Figure adapted from [40].)

The balloon force is combined with the GVF forces in order to overcome the limitation of the leakage of the contour close to the weak edges of interest. The weighted external energy was proposed to evolve the contour based on the intensity of the pixels of the edge map ahead the points of the initial contour. The balloon force is employed here to increase the amplitude of the gradient vectors to accelerate the expansion of the contour towards the border of interest. However, it tends to fail when a homogeneous region is found or the gradient forces that surround the contour to be evolved are weak enough to stop the expansion or contraction in the border of interest. Hence, the balloon force in Equation 4 has effect on the expansion of the contour only when the intensities of the pixels of the edge map ahead the contour are homogeneous or the gradient forces measured from the GVF are strong enough to allow the contour to be evolved. Given an edge map image $f$ obtained by a gradient operator with edges represented by high intensity values and non-edge regions represented by low intensity values, the main idea of the external energy proposed by Khadidos et al. [40] is to apply the balloon forces to continue the evolution of the contour when a homogeneous region with 
low intensities is found. On the contrary, when an edge is found, the GVF based force is applied to fit the

contour to this edge.

The weighted external energy was proposed to force the expansion of the contour in homogeneous regions, and attenuate the evolution in heterogeneous regions close to the desired boundary. However, the leakage of the contour remains a major challenge due to low intensity values in regions with weak edges. Since the average value $h$ of the intensities inside the semi-circular region tends to zero close to weak edges, the balloon forces predominate the external energy proposed in Equation 4 , leading the contour to leak the true boundary of the region of interest. Hence, a modification of the weighted external energy defined in Equation 4 is proposed to improve the segmentation results in weak boundaries. The modification consists in replacing average value $h$ by the entropy of the intensity values ahead pixel $(x, y)$ of the contour to be deformed by:

$$
h(x, y)=1-\frac{1}{\text { entropy }(P)+1},
$$

where entropy $(P)$ is the entropy of the pixels inside the semi-circle centred at contour pixel $P$ with coordinates $(x, y)$ :

$$
\text { entropy }=-\sum_{i=1}^{N} p_{i} * \log _{2}\left(p_{i}\right),
$$

$N$ is the highest possible intensity value, and $p_{i}$ is the probability of occurrence of intensity value $i$. An entropy close or equal to 0 (zero) represents a homogeneous region; whereas an entropy greater than 0 (zero), indicates a heterogeneous region. Hence, in a homogeneous region, the value of $h$ tends to 0 (zero) and the balloon force is prevalent on the weighted external energy. On the other hand, the value of $h$ tends to 1 (one)

when the region is heterogeneous, leading to the application of the GVF in Equation 4 . Unlike the average of the grayscale intensities, the entropy is more sensitive to the variation of intensities inside the region ahead the pixel of the contour to be deformed. Hence, the GVF in Equation 4 tends to be applied in regions with high entropy, leading the contour to stop in weak boundaries. Besides the use of the entropy, a weighting factor is proposed to decrease the evolution of the contour when regions with low intensities are found:

$$
A(x, y)=\frac{1}{N} \sum_{i, j \in S} f(i, j)
$$

255 where $N$ is the number of pixels inside semi-circular region $S$, and $(x, y)$ is a pixel of the contour. In BlackBlood MR images, the region outside the vessel wall is composed of low intensity values. Therefore, the proposed weighting factor attenuates the evolution in regions with low intensities, while maintaining the evolution in regions with high intensity pixels. The GVF in Equation 4 was replaced by the NNGVF to handle more efficiently regions corrupted by noise, and the following modified weighted external energy is proposed to find the boundaries of carotid arteries:

$$
E_{\text {ext }}=A *\left(\left(F_{\text {balloon }} *(1-\Omega)\right)+\left(F_{N N G V F} * \Omega\right)\right)
$$


where $A$ is the weighting factor calculated for each contour pixel as defined in Equation 10, Regions $S$ and $T$ used to calculate the values of $A D$ and $h$ defined in Equations 7 and 8 were defined as semi-circular shaped regions to improve the capture range of the pixels ahead the pixel of the contour to be expanded towards the boundary of the carotid artery.

$\left(c_{x}, c_{y}\right)$ is measured by taking the mean of coordinates $x$ and $y$ of the contour, respectively. The value of $\theta_{m}$ is obtained by calculating the angle between the major axis and the $\mathrm{x}$-axis represented by the straight arrow in Figure 6. The value of $\theta_{i}$ is obtained from the calculated angle between contour point $\left(x_{i}, y_{i}\right)$ and the major axis. The contour of the lumen might be irregular for calculating the major and minor axis based on 
285 Equation 12. However, the ellipse constraint was proposed in order to correct irregularities on the contour based on distances between the contour points and the major and minor axis, leading the contour to fit an ellipse-shaped model. If the distance between a certain point $\left(x_{i}, y_{i}\right)$ of the contour is far away from the estimated circle measured by centroid $\left(c_{x}, c_{y}\right)$ and the estimated major and minor axes (as illustrated by the dotted curve in Figure 6), the point is corrected to fit the estimated ellipse-shaped contour. After all the points of the contour be corrected, the Snake model is applied again to deform the new ellipse-shaped contour.

Therefore, Equation 12 is integrated into the constraint of the used Snake model, which is applied to the images resulting from the gray-weighted distance proposed in Equation 1 to expand the lumen contour beyond the boundaries of the lumen region. Examples of the expansion of the lumen contour performed by the used Snake model with the ellipse constraint are shown in Figures 7 and 8
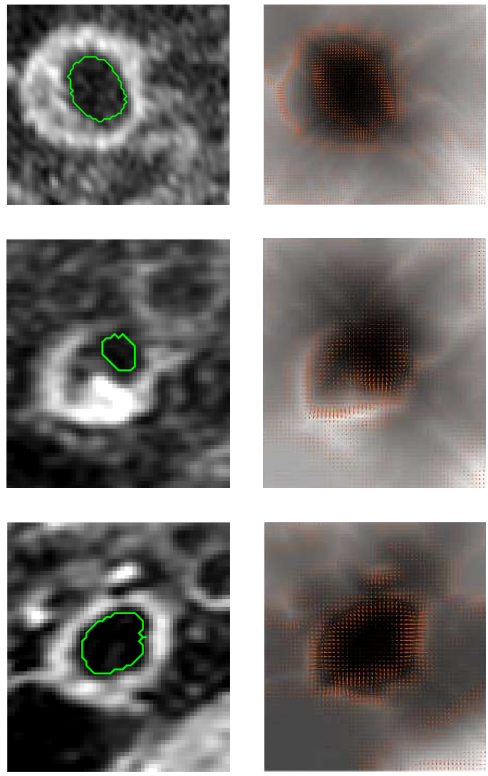

(a)

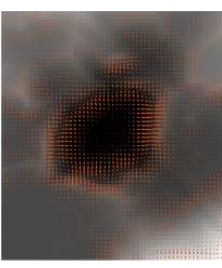

(b)
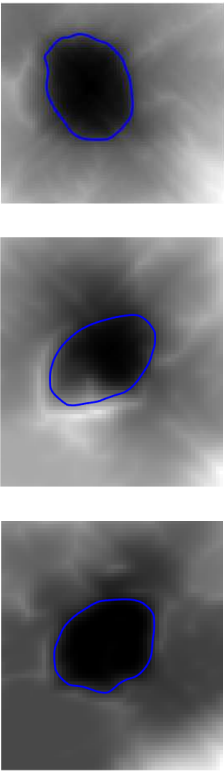

(c)
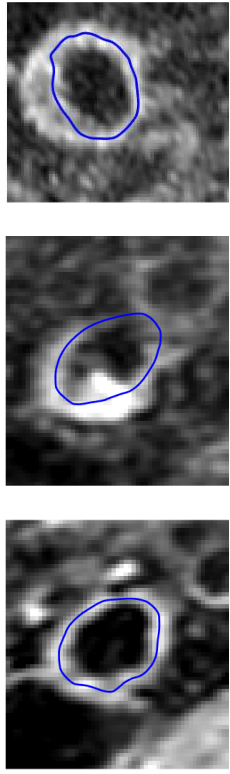

(d)

Figure 7: Examples of the expansion of lumen contours resulting from the used Snake model with the ellipse constraint applied to images obtained based on the gray-weighted distance: a) Original MR images with the segmented lumen contours in green; b) The gradient vectors (in orange) computed from the images obtained based on the gray-weighted distance; c) The expanded lumen contours (in blue) overlapped on the images representing the distance maps; d) The expanded lumen contours overlapped on the original MR images.

As shown in Figure 7 , the lumen and carotid wall boundaries in images representing the distance maps progressively increase the magnitudes and convergence of the gradient vectors. This behaviour is due to the fact that the gradient magnitude of the distance map image is proportional to the distance value associated to each pixel [43]. Therefore, the magnitude of the gradient vectors tends to increase at regions with higher distances calculated with respect to the centre of the lumen region, leading the contour to expand beyond the boundary of the lumen region, as shown in Figure $7 \mathrm{~b}$. 

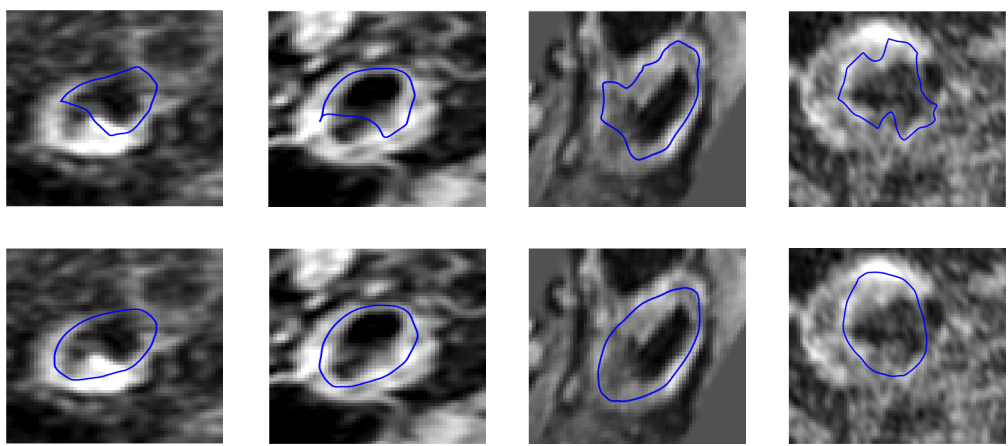

Figure 8: Examples of the expansion of lumen contours performed by the used Snake model without (images on the top row) and with (images on the bottom row) taking into account the ellipse constraint.

In Figure 8 the contours produced by the Snake model without taking into account the ellipse constraint were trapped due to the grayscale intensity variations inside the carotid wall. Particularly, the examples shown in the images on the top row of Figure 8 depict incomplete evolutions of the contours caused by gradient vectors with high magnitudes in the low intensities regions close to the lumen boundary. However, after the employment of the ellipse constraint, the contours overcome the regions with high intensity variations and assume more regular shapes.

\subsection{Validation measures}

The contours segmented by the proposed approach and the corresponding manual delineations were compared based on four measures: Dice coefficient (DC), Polyline distance (PD), mean contour distance (MCD) and centroid distance (CD). The Dice coefficient is used to calculate the overlap between the automatic $\left(S_{\text {auto }}\right)$ and manual $\left(S_{\text {manual }}\right)$ segmentations:

$$
D C=\frac{2 *\left|S_{\text {auto }} \cap S_{\text {manual }}\right|}{\left|S_{\text {auto }}\right|+\left|S_{\text {manual }}\right|} .
$$

The Polyline distance represents the average minimum distance between two sets of points, i.e., image pixels, and indicates how far one set is from the other one, being calculated as:

$$
D_{s}\left(B_{1}, B_{2}\right)=\frac{d\left(B_{1}, B_{2}\right)+d\left(B_{2}, B_{1}\right)}{N\left(B_{1}\right)+N\left(B_{2}\right)}
$$

where $B_{1}$ and $B_{2}$ are the two sets of points under comparison, $N\left(B_{1}\right)$ and $N\left(B_{2}\right)$ are the number of points in $B_{1}$ and $B_{2}$, respectively, and $d\left(B_{1}, B_{2}\right)=\sum_{v \in B_{1}} \min \{d(v, s)\}$ and $d\left(B_{2}, B_{1}\right)=\sum_{s \in B_{2}} \min \{d(s, v)\}$.

An under- and over-estimation of the segmented region may affect the values calculated by the Dice coefficient. However, the difference between the centroid of the contour segmented by the proposed approach and the centroid of the corresponding manual delineation is low when the approach finds the correct location of the region. Therefore, the centroid difference was also employed according to:

$$
C D=\sqrt{\left(x_{s}-x_{g}\right)^{2}+\left(y_{s}-y_{g}\right)^{2}}
$$


where $\left(x_{s}, y_{s}\right)$ and $\left(x_{g}, y_{g}\right)$ represent the centroids of the segmented region and of the corresponding manual delineation, respectively. The higher the centroid distance, the greater the distance between the regions.

The lumen area, total carotid area, average lumen diameter, average carotid diameter and vessel wall thickness of the automatic and manual segmentations of the lumen and carotid artery contours were also calculated to compare the segmentation results by means of the linear regression analysis and Bland-Altman analysis. The lumen area and the total carotid area are the absolute areas inside the contours of the lumen and carotid artery, respectively, and the average diameter (AVD) of the segmented contours is defined as:

$$
A V D=\frac{1}{N} \sum_{i=1}^{N} 2 * r_{i},
$$

where $N$ is the number of pixels of the contour and $r_{i}$ is the radius of pixel $i^{t h}$, which is multiplied by 2 in order to calculate the diameter of the contour at pixel $i^{t h}$. The vessel wall thickness is defined as the mean distance between the segmented contours of the lumen and carotid artery.

The Wilcoxon signed-rank test with $5 \%$ of significance was further applied to the obtained results in order to conduct the statistical analysis.

\section{Experimental results}

A comparison between the contours obtained by the proposed approach and the corresponding ones generated by manual delineations was performed in order to evaluate the accuracy of the segmentation results.

\subsection{Parameters initialization}

To segment the boundary of each carotid artery in the experimental image dataset, the proposed approach was used with the parameter values indicated in Table 1 .

Table 1: Values of the parameters used in the segmentation of the carotid artery boundaries under study.

\begin{tabular}{lrr}
\hline Parameter & 1st step & 2nd step \\
\hline$\alpha$ & 0.05 & 0.05 \\
$\beta$ & 0 & 1 \\
$\mu$ & 0.02 & 0.02 \\
$\kappa$ & 1 & 1 \\
$\sigma$ & 1 & 1 \\
Radius of the semi-circle & 5 & 5 \\
Number of iterations & 150 & 500 \\
\hline
\end{tabular}

The value of parameter $\alpha$ was set to 0.05 to avoid the contour to become too rigid in regions with large areas. The value of $\beta$ was set to 1 (one) in the second step of the proposed approach in order to the final contour of the carotid artery be smoother. The value of $\kappa$ was also set to 1 (one) to avoid the excessive 
amplitude of the balloon force defined in Equation 3. The value of $\sigma$ was set to 1 (one) to avoid the excessive smoothing of the boundaries of interest and consequently, the weakening and displacement of the gradient and 8 were defined experimentally by combining and testing a set of values over 40 images of three patients randomly selected. In this tuning process, the values chosen for $\mu$ varied from 0.01 to 0.07 according to increments of 0.01 , whereas the values selected for the radius of the semi-circle were between 2 and 10 . The mean Polyline distance was calculated for each combination of the values of $\mu$ and the radius of the semifor the images of the three patients, were chosen to be the best values for the dataset under study. Due to the computational cost required by the ellipse constraint, the number of iterations used in the first step of the proposed approach was lower than the one used in the second step. However, the value chosen was able to successfully expand the lumen contour beyond the boundary of the lumen region. Table 2 presents the average values of the Polyline distance obtained for each combination of parameter $\mu$ with the radius of the semi-circle. The average values found for the Dice coefficient, Polyline distance, mean contour distance and centroid distance that led to the best combination of $\mu$ and the radius of the semi-circle are indicated in Table 3 .

Table 2: Average values of the Polyline distance (in pixels) for each combination of parameter $\mu$ and the radius of the semicircular region obtained from 40 images. (The value in bold is the minimum average Polyline distance obtained for the images of the three patients used for tuning these parameters.)

\begin{tabular}{lrrrrrrrrrrr}
\hline & Radius & $\mathbf{2}$ & $\mathbf{3}$ & $\mathbf{4}$ & $\mathbf{5}$ & $\mathbf{6}$ & $\mathbf{7}$ & $\mathbf{8}$ & $\mathbf{9}$ & $\mathbf{1 0}$ \\
\hline $\mathbf{0 . 0 1}$ & & & & & & & & & & \\
$\mathbf{0 . 0 2}$ & 4.0117 & 2.8806 & 2.6332 & 2.4892 & 2.5602 & 2.5722 & 2.6396 & 2.7048 & 2.7265 \\
$\mathbf{0 . 0 3}$ & 3.4291 & 2.6974 & 2.5738 & $\mathbf{2 . 4 6 7 5}$ & 2.598 & 2.7162 & 2.7595 & 2.8254 & 2.8547 \\
$\mathbf{0 . 0 4}$ & 3.203 & 2.6762 & 2.5468 & 2.5519 & 2.7642 & 2.8216 & 2.8431 & 2.9811 & 3.0341 \\
$\mathbf{0 . 0 5}$ & 3.0366 & 2.6938 & 2.5171 & 2.7535 & 2.9772 & 3.1496 & 3.1824 & 3.1831 & 3.327 \\
$\mathbf{0 . 0 6}$ & 2.9615 & 2.5438 & 2.6402 & 2.9168 & 3.1949 & 3.3402 & 3.4034 & 3.5569 & 3.6093 \\
$\mathbf{0 . 0 7}$ & 2.717 & 2.7496 & 2.9286 & 3.9539 & 3.4282 & 3.6216 & 3.6871 & 3.7895 & 3.8347 \\
\hline
\end{tabular}

Table 3: Average values found for the Dice coefficient, Polyline distance, mean contour distance and centroid distance that led to the best combination of $\mu$ and the radius of the semi-circle.

\begin{tabular}{lcr}
\hline Validation measure & Weighted external energy & NNGVF \\
\hline Dice coefficient & $0.8625 \pm 0.0863$ & $0.7666 \pm 0.1202$ \\
Polyline distance (px) & $2.4675 \pm 1.7135$ & $4.001 \pm 2.7791$ \\
Mean contour distance (px) & $2.4561 \pm 1.6779$ & $3.2355 \pm 1.5508$ \\
Centroid distance $(\mathbf{p x})$ & $2.9309 \pm 2.6268$ & $5.2198 \pm 2.9887$ \\
\hline
\end{tabular}

*NNGVF=Neighbourhood-Extending and Noise-Smoothing Gradient Vector Flow; px=pixels. 


\subsection{Performance evaluation}

Examples of carotid artery boundaries segmented by the proposed approach and the corresponding manual delineations are shown in Figure 9 .
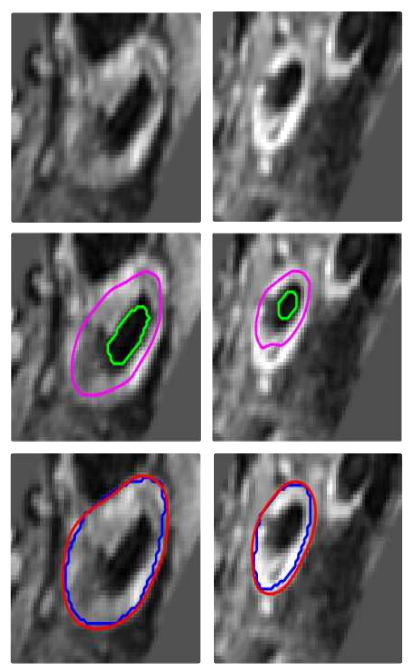
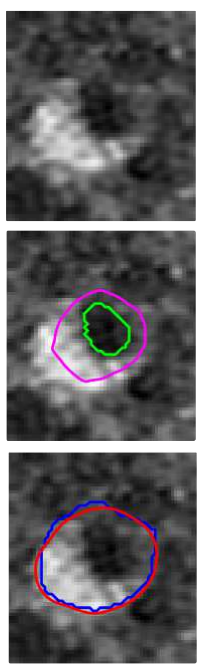
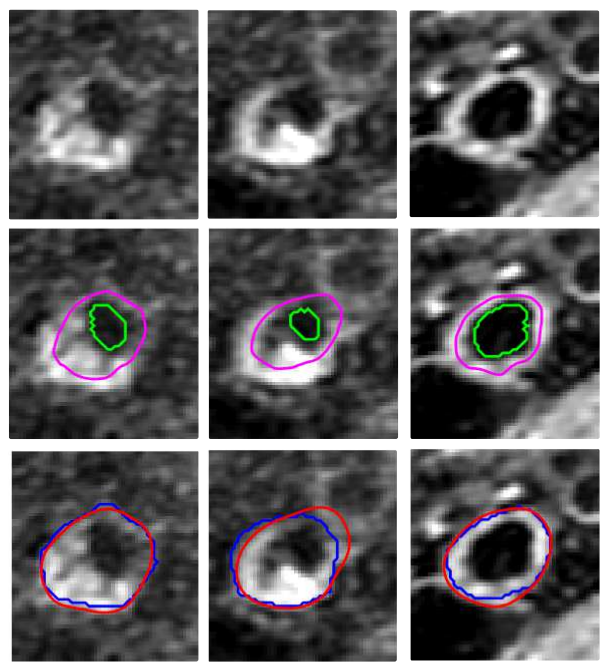

Figure 9: Examples of carotid artery boundaries segmented by the proposed approach: the original PDW MR images are shown on the top row; the images in the middle row illustrate the lumen contours (in green) along with the contours expanded based on the images obtained by the gray-weighted distance (in magenta), which were then used to initiate the segmentation of the carotid artery boundaries; the images on the bottom row illustrate the carotid artery boundaries segmented by the Snake model with the proposed weighted external energy (in red) and the corresponding manual delineations (in blue).

The images in the middle row of Figure 9 show the lumen contours in green obtained by the automatic method proposed in Jodas et al. 25] for the segmentation of the lumen region, whereas the ones obtained from these contours by their expansion, using the Snake model with the ellipse constraint on the images obtained by the gray-weighted distance, are in magenta. In the bottom row of the same figure, the contours in red are the carotid artery boundaries found by the Snake model with the proposed weighted external energy, and the contours in blue are the corresponding manual delineations. From the images shown, it is possible to notice that the carotid artery boundaries were correctly segmented in all images, with the automated results very close to the corresponding manual delineations.

The comparison performed between the carotid artery boundaries obtained by the Snake model with the proposed weighted external energy and those generated by only taking into account the forces calculated by the NNGVF, as well as the boundaries obtained by the method proposed by [40], is depicted in Figure 10,

The images in the middle row of Figure 10 show that the proposed approach is effective to expand the lumen contour to the true boundary of the carotid artery. The incomplete evolution of the lumen contour when only the forces calculated by the NNGVF are used was mainly due to the large thickness and grayscale intensity variations inside the wall of the carotid artery under study. Besides, the modified weighted external energy was also more efficient than the one proposed by [40] in handling the regions where the border is weak, as shown in Figures 10(a-c). The only exception is the example illustrated by Figure 10(d), where the 

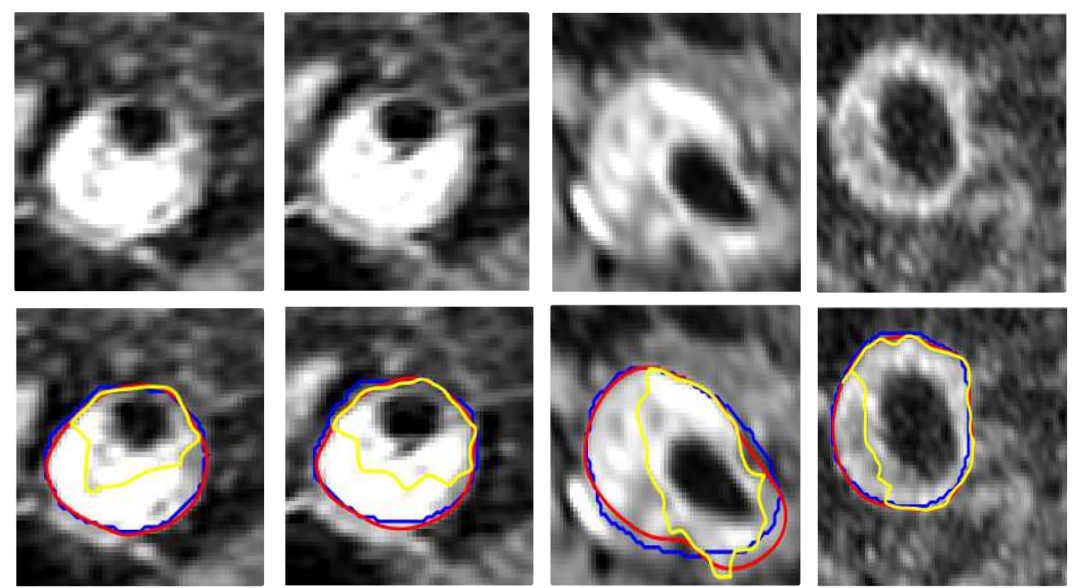

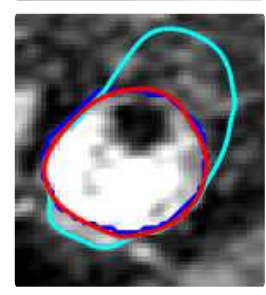

(a)

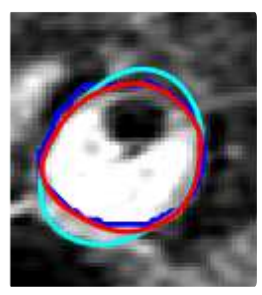

(b)

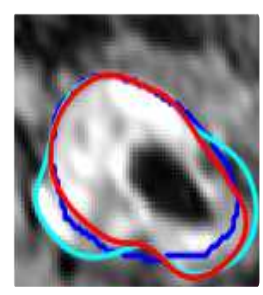

(c)

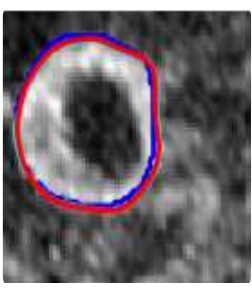

(d)

Figure 10: Examples of carotid artery boundaries obtained by the Snake model with the proposed weighted external energy (in red) and the ones generated by only taking into account the forces calculated by the NNGVF (in yellow) and the weighted external force proposed by [40] (in cyan). (The original PDW MR images are shown on the top row; the corresponding manual delineations are shown in blue.)

contour obtained by the external energy proposed by [40] was quite similar to the contour obtained by our modified proposed external energy.

Regarding the quantitative analysis, the Dice coefficient values for the carotid artery boundaries obtained by the Snake model with the proposed weighted external energy (in red) and the corresponding manual delineations (in blue) shown in the second row of Figure 10 were equal to 0.97, 0.96, 0.93 and 0.97, respectively. On the other hand, the Dice coefficient values for the Snake model with the NNGVF (in yellow) and the corresponding manual delineations (in blue) were equal to $0.65,0.72,0.71$ and 0.87 , respectively. Considering the third row of Figure 10, the Dice coefficient values obtained taking into account the Snake model with the weighted external energy proposed by [40] (in cyan) and the corresponding manual delineations (in blue) were equal to $0.77,0.89,0.89$ and 0.97 , respectively. The distribution of the Dice coefficient values calculated from the segmented lumen and carotid artery regions and the corresponding manual delineations are depicted according to intervals of 0.2 in Figures 11 and 12, respectively.

In terms of the lumen segmentation results, the interval of the Dice coefficient values between 0 (zero) and 0.20 includes the images erroneously segmented. From the 185 PDW MR images used in the experiments, the lumen region was successfully segmented in 139 images. As depicted in Figure 11 the images with the Dice coefficient values between 0 (zero) and 0.20, represent 26\% (49 images) of the total segmentation results. 


\section{Number of images per interval of Dice coefficent}

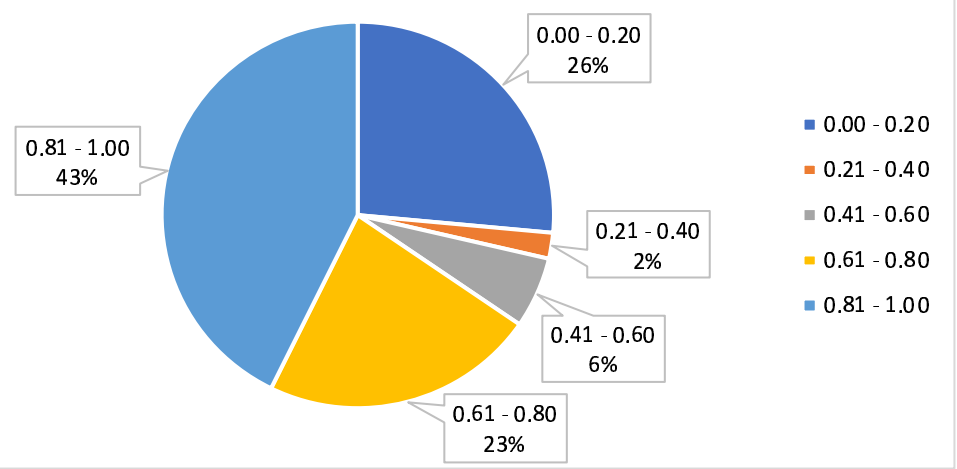

Figure 11: Intervals of the Dice coefficient values calculated from the segmented lumen regions and the corresponding manual delineations.

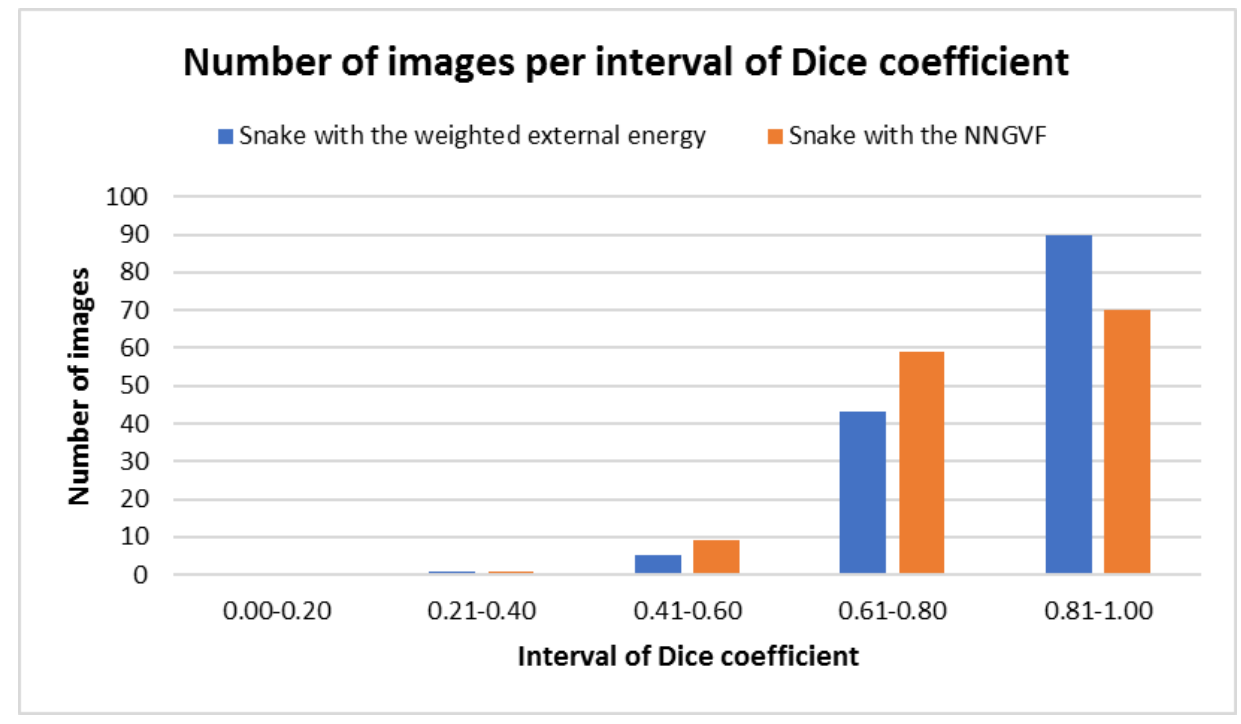

Figure 12: Intervals of the Dice coefficient values calculated from the segmented carotid artery regions and the corresponding manual delineations.

The images with the Dice coefficient values from 0.21 to 0.40 (4 images) and from 0.41 to 0.60 (11 images), represent only $2 \%$ and $6 \%$, respectively, of the total segmented lumen regions. Contrary, the resultant images with the Dice coefficient values from 0.61 to 0.80 (42 images) and from 0.81 to 1.00 (79 images), represent $23 \%$ and $43 \%$ of the total segmented images, respectively, reaching in total the greatest percentage of the total number of images used in the experiments. Regarding the segmentation of the carotid artery boundaries, the number of images having the Dice coefficient value between 0.81 and 0.90 is higher for the Snake model with the proposed weighted external energy in comparison with the Snake model using only the forces calculated by the NNGVF. As shown in Figure 12, 90 images with a Dice coefficient value between 0.81 and 1.00 were successfully segmented. On the other hand, the number of images with a Dice coefficient value in the same 
interval decreased to 76 when the Snake model with only the NNGVF forces was employed.

The average values found for the Dice coefficient, Polyline distance, mean contour distance and centroid distance of the segmented lumen contours in comparison with the corresponding manual delineations are indicated in Table 4. Additionally, the average values of the same quantitative measures calculated from the segmented carotid artery boundaries and the corresponding manual delineations are indicated in Table [5 including the measurements obtained from the segmentation results performed by the weighted external ${ }_{410}$ energy proposed by [40].

Table 4: Average values found for the Dice coefficient, Polyline distance (PD), mean contour distance (MCD) and centroid distance $(\mathrm{CD})$ concerning the lumen segmentation.

\begin{tabular}{lcrrr}
\hline & Dice \pm std & PD \pm std $(\mathbf{p x})$ & $\mathbf{M C D} \pm \mathbf{s t d}(\mathbf{p x})$ & $\mathbf{C D} \pm \mathbf{s t d}(\mathbf{p x})$ \\
\hline Patient 1 & $0.86 \pm 0.06$ & $1.50 \pm 0.57$ & $1.39 \pm 0.51$ & $1.95 \pm 1.03$ \\
Patient 2 & $0.71 \pm 0.17$ & $2.65 \pm 1.26$ & $2.60 \pm 1.70$ & $3.30 \pm 2.51$ \\
Patient 3 & $0.81 \pm 0.17$ & $1.24 \pm 1.28$ & $0.77 \pm 0.50$ & $1.49 \pm 1.88$ \\
Patient 4 & $0.48 \pm 0.21$ & $2.24 \pm 0.30$ & $1.63 \pm 0.67$ & $1.63 \pm 0.05$ \\
Patient 5 & $0.82 \pm 0.10$ & $0.78 \pm 0.27$ & $0.69 \pm 0.18$ & $0.96 \pm 0.42$ \\
Patient 6 & $0.75 \pm 0.15$ & $2.51 \pm 2.81$ & $2.62 \pm 3.62$ & $3.24 \pm 4.92$ \\
Patient 7 & $0.78 \pm 0.11$ & $1.16 \pm 0.29$ & $1.11 \pm 0.37$ & $1.55 \pm 0.63$ \\
Patient 8 & $0.83 \pm 0.12$ & $1.15 \pm 0.59$ & $1.18 \pm 0.68$ & $1.22 \pm 0.74$ \\
Patient 9 & $0.73 \pm 0.22$ & $1.14 \pm 0.67$ & $1.03 \pm 0.85$ & $1.45 \pm 0.95$ \\
Patient 10 & $0.69 \pm 0.22$ & $1.81 \pm 2.38$ & $1.90 \pm 2.99$ & $2.03 \pm 3.32$ \\
Patient 12 & $0.69 \pm 0.21$ & $2.07 \pm 2.60$ & $2.09 \pm 3.14$ & $2.43 \pm 3.15$ \\
\hline Average & $0.76 \pm 0.17$ & $1.49 \pm 1.53$ & $1.40 \pm 1.81$ & $1.80 \pm 2.20$ \\
\hline
\end{tabular}

${ }^{*} \mathrm{px}=$ pixels; std $=$ standard deviation.

Table 5: Averages values found for the Dice coefficient, Polyline distance (PD), mean contour distance (MCD) and centroid distance $(\mathrm{CD})$ regarding the segmentation of the carotid artery boundary.

\begin{tabular}{lrrr}
\hline Validation measure & PWED & NNGVF & WED \\
\hline Dice coefficient & $0.83 \pm 0.11$ & $0.80 \pm 0.11$ & $0.74 \pm 0.13$ \\
Polyline distance (px) & $2.70 \pm 1.69$ & $3.01 \pm 2.05$ & $4.76 \pm 2.85$ \\
Mean contour distance (px) & $2.79 \pm 1.89$ & $2.87 \pm 1.67$ & $11.88 \pm 5.55$ \\
Centroid distance (px) & $3.44 \pm 2.82$ & $4.18 \pm 2.97$ & $4.65 \pm 2.74$ \\
Error ratio of segmentation & $0.13 \pm 0.11$ & $0.11 \pm 0.09$ & $0.17 \pm 0.12$ \\
\hline
\end{tabular}

*NNGVF=Neighbourhood-Extending and Noise-Smoothing Gradient Vector Flow; px=pixels

*PWED = Proposed Weighted External Energy; WED = Weighted External Energy proposed by [40].

In terms of the lumen segmentation results, the average values of the Dice coefficient obtained for patient 1 are higher than the ones obtained for the other patients, as indicated in Table 4 . On the other hand, the averages values of the Polyline, mean contour and centroid distances are lower for patient 5 relatively to the averages values for the other patients. As indicated in Table 5 the results obtained using the Snake model with the proposed weighted external energy for the segmentation of the carotid artery boundary are 
better than the majority obtained using the Snake model with the NNGVF and the weighted external energy proposed by [40]. Regarding the average values of the validation measures, the Dice coefficient increased to $0.83 \pm 0.11$ when the proposed weighted external energy was used in the Snake model. The averages values of the Polyline distance decreased to $2.70 \pm 1.69$ pixels after the usage of the Snake model with the proposed weighted external energy. Additionally, the average value of the centroid distance obtained from the proposed approach is lower than the ones obtained from the Snake model with the NNGVF and the weighted external energy proposed by [40] ( $3.44 \pm 2.82$ pixels against $4.18 \pm 2.97$ and $4.65 \pm 2.74$, respectively).

The Linear regression and Bland-Altman analysis concerning the lumen area and the average lumen diameter showed a good correlation between the segmented lumen contours and the corresponding manual delineations, as can be observed in Figure 13 The linear regression and Bland-Altman analysis for the total carotid area, average carotid diameter and vessel wall thickness calculated concerning the segmented carotid artery boundaries and the corresponding manual delineations are depicted in Figures 14 and 15 .

As shown in Figure $13 \mathrm{k}$, the Spearman correlation between the segmented lumen contours and the corresponding manual delineations was high and similar for both measures, reaching a value of 0.8811 and 0.8764 for the lumen area and average lumen diameter, respectively. For the segmentation of the carotid artery boundaries, the Spearman correlation between the total carotid areas obtained using the Snake model with the proposed weighted external energy and the corresponding areas generated from the manual delineations was equal to 0.7956 , which is higher than the correlation coefficient of the segmentation results obtained by the Snake model with the NNGVF (0.7338). Regarding the average carotid diameter, the Spearman correlation between the segmentations obtained by the proposed approach and the corresponding manual delineations was equal to 0.7974 , which is also better than the one obtained from the comparison between the ones of the Snake model with the NNGVF and the manual delineations (0.7333). Additionally, the Spearman correlation between the proposed approach and the corresponding manual delineations showed also better results in comparison to the Snake model with the NNGVF regarding the vessel wall thickness (0.6308 and 0.6041, respectively). The differences between the segmented lumen contours and the corresponding manual delineations are shown in the Bland-Altman plots of Figure 13b. The Bland-Altman analysis of the carotid artery boundaries segmented by the Snake model with the proposed weighted external energy and NNGVF are depicted in Figures 14b and 15b, respectively. Regarding the lumen area, the average difference between the segmented lumen contours and the corresponding manual delineations was equal to 13.50 pixels. For the average lumen diameter, the average difference between the segmented and manually delineated lumen contours was equal to 0.21 pixels. For the total carotid area and average carotid diameter, the average differences between the proposed approach and manual delineations were of 90 and 1.8 pixels, respectively. The average difference between the vessel wall thickness calculated from the carotid artery boundaries segmented by the proposed approach and the ones calculated from the corresponding manual delineations was equal to 1.2 pixels. The high average difference of the total carotid area was due to the similar intensities in regions close to the boundaries of the carotid arteries, which makes more difficult the distinction between 


\section{Lumen area}
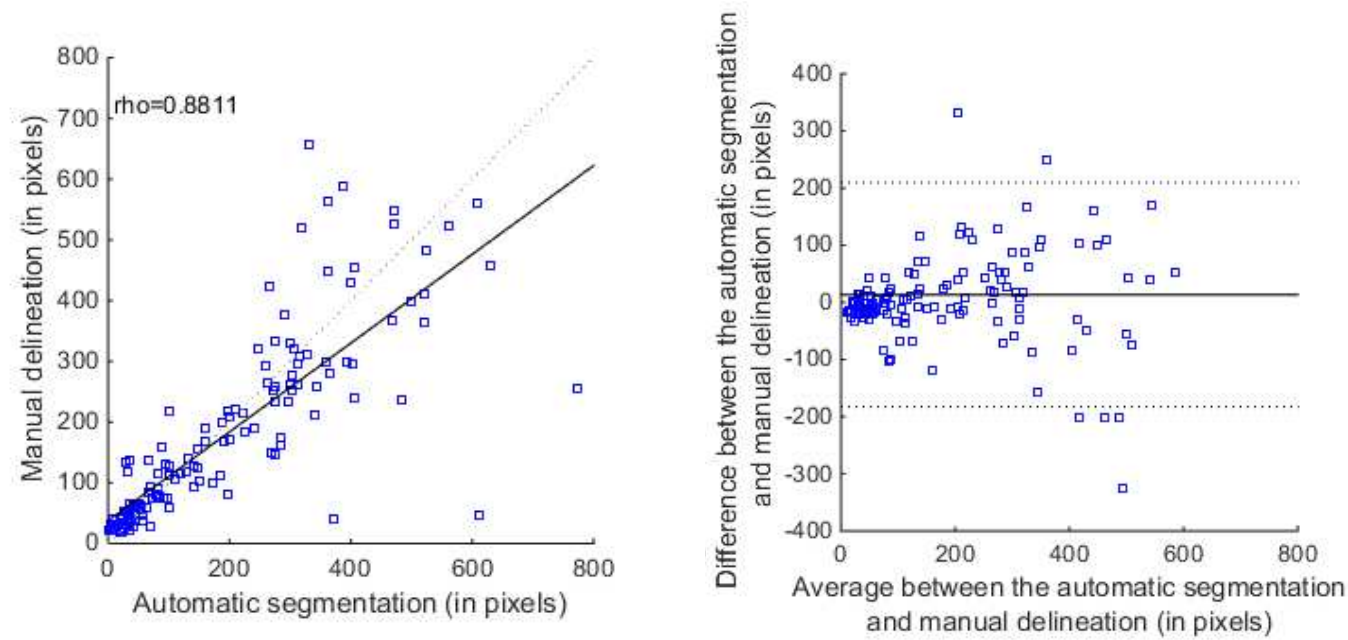

\section{Average lumen diameter}

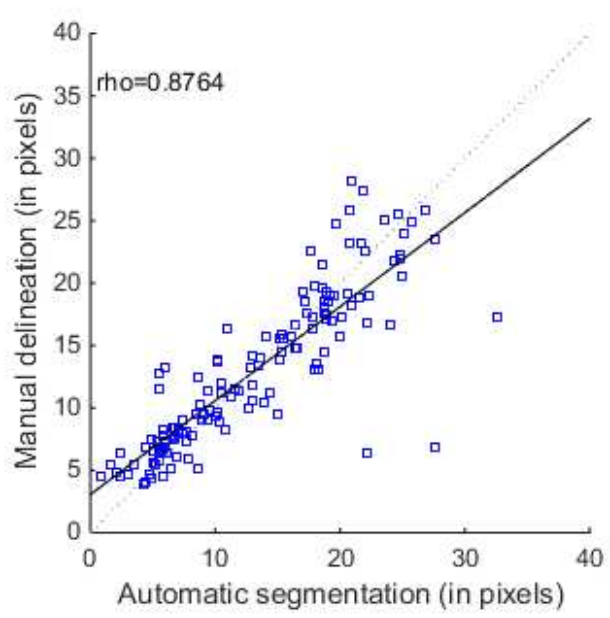

(a)

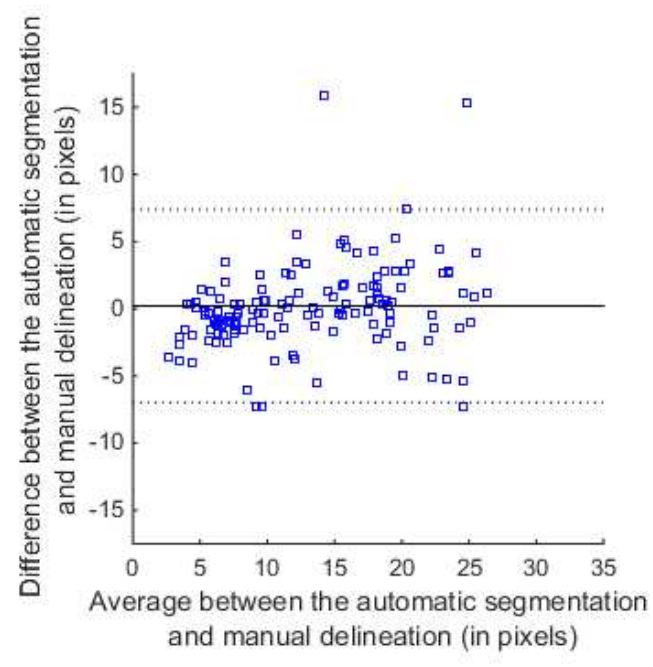

(b)

Figure 13: Linear regression (a) and Bland-Altman analysis (b) concerning the lumen area and average lumen diameter calculated from the segmented lumen contours and the corresponding manual delineations.

the carotid boundaries and the image background. Examples of carotid artery boundaries segmented by the proposed approach in images with background regions having intensities similar to the ones of the carotid artery boundaries are illustrated in Figure 16

As depicted in Figure 16, the carotid artery boundaries segmented by the Snake model with the proposed weighted external energy overestimated the corresponding manual delineations due to the similar grayscale intensities surrounding the true boundaries of interest, leading to an increased difference between the areas of the automatically segmented and manually delineated carotid artery boundaries.

Table 6 presents $p$-values calculated from the Wilcoxon signed-rank test as a statistical tool to check 


\section{Total carotid area}
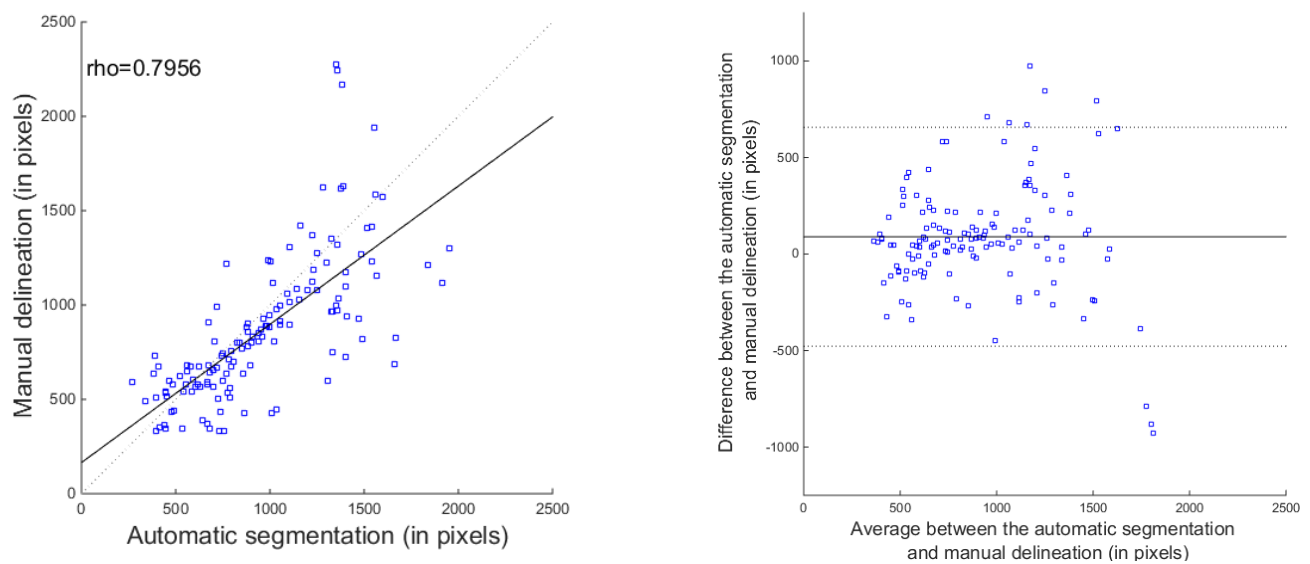

Average carotid diameter
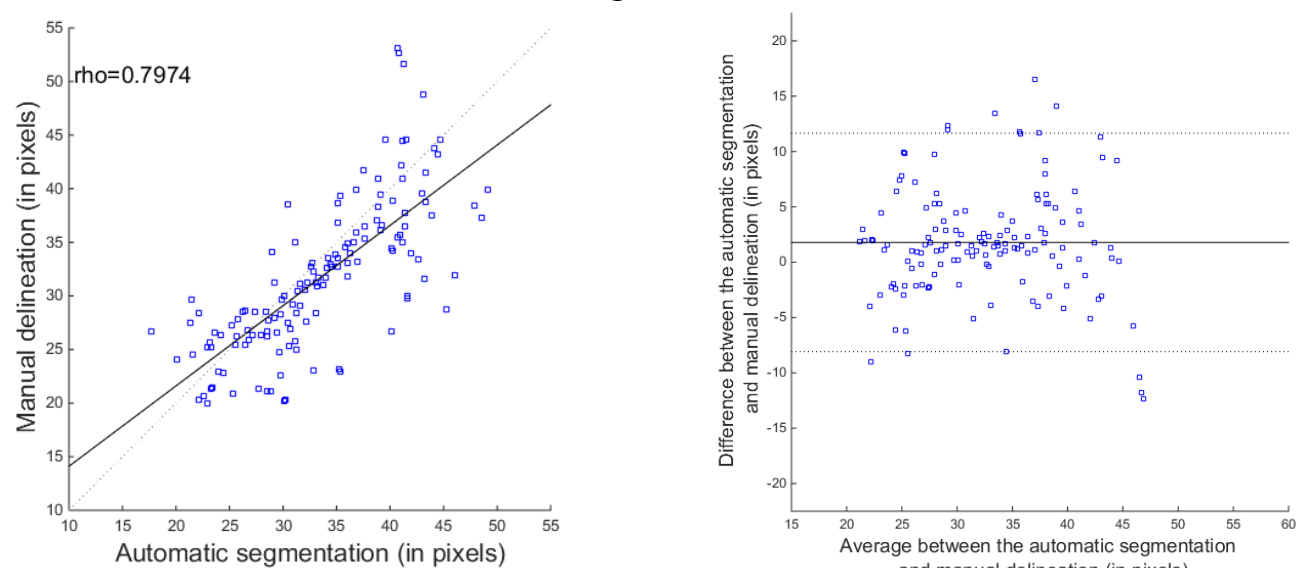

and manual delineation (in pixels)

Vessel wall thickness

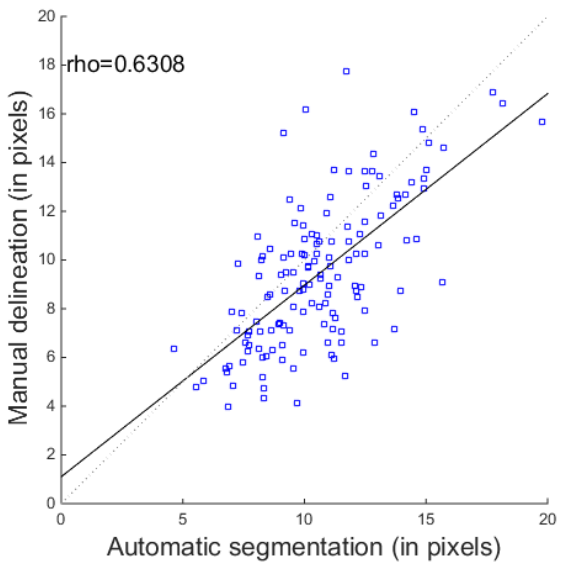

(a)

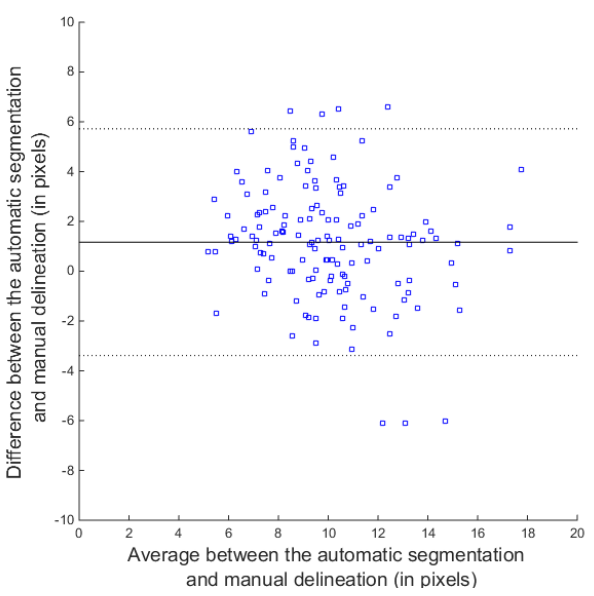

(b)

Figure 14: Linear regression (a) and Bland-Altman analysis (b) concerning the total carotid area, average carotid diameter and vessel wall thickness calculated from the carotid artery contours segmented by the Snake model with the proposed weighted external energy and the corresponding manual delineations. 


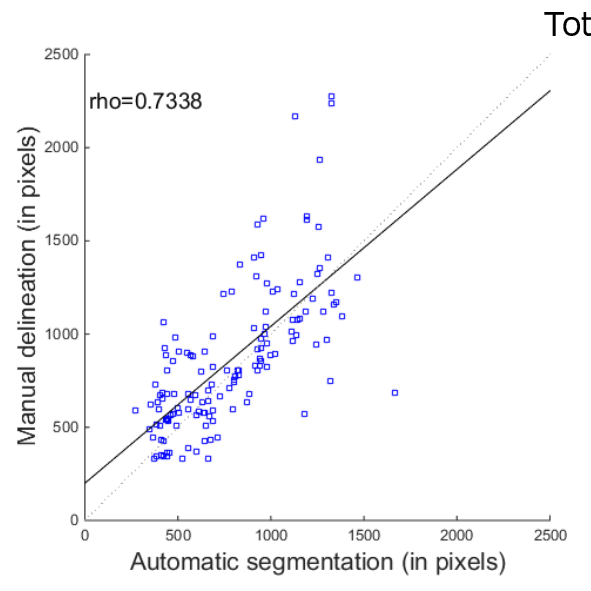

Total carotid area

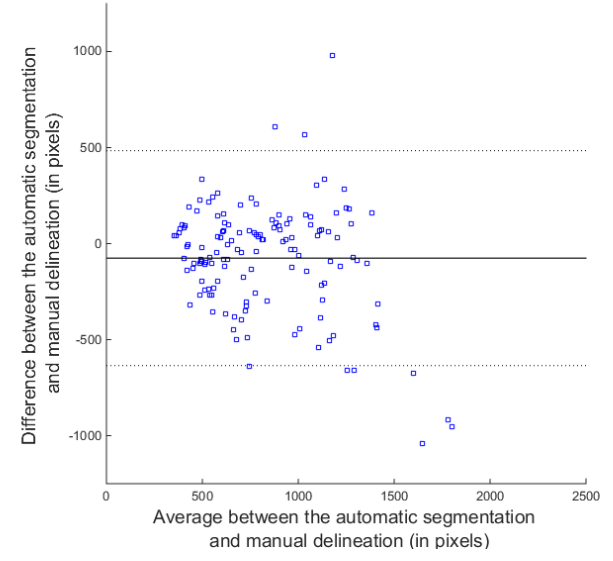

Average carotid diameter
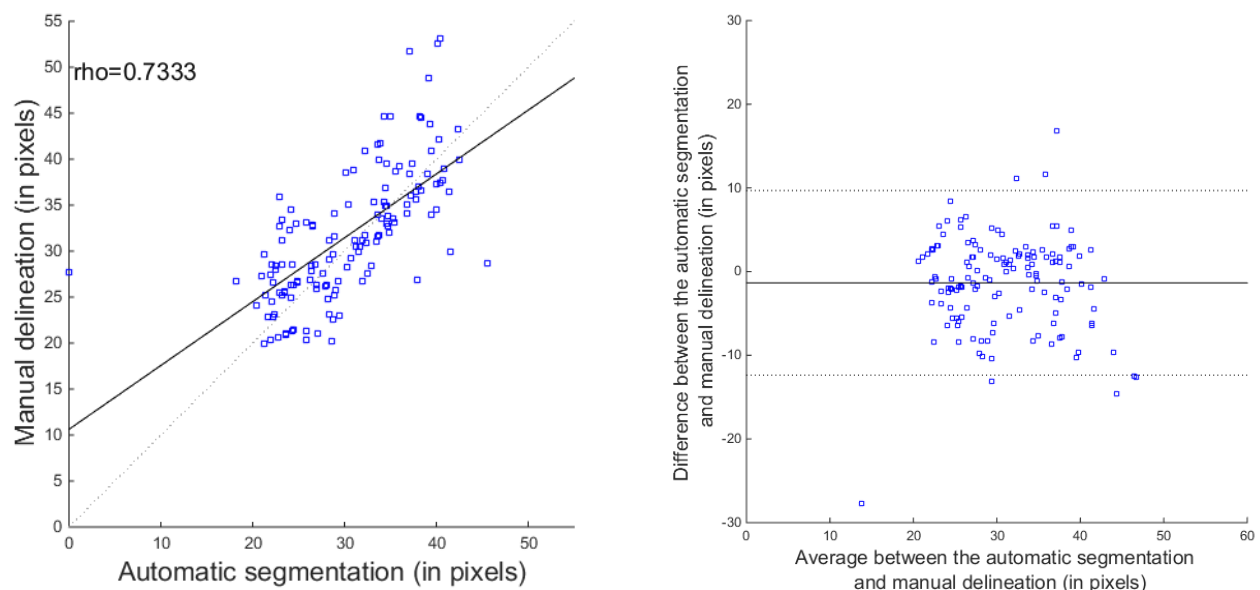

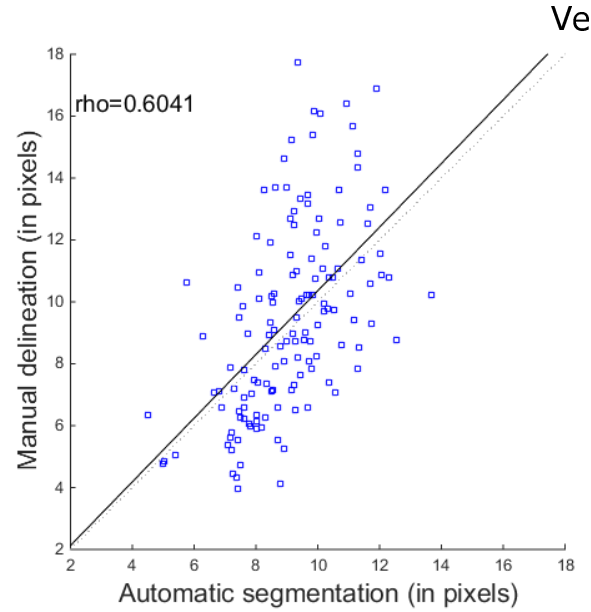

(a)

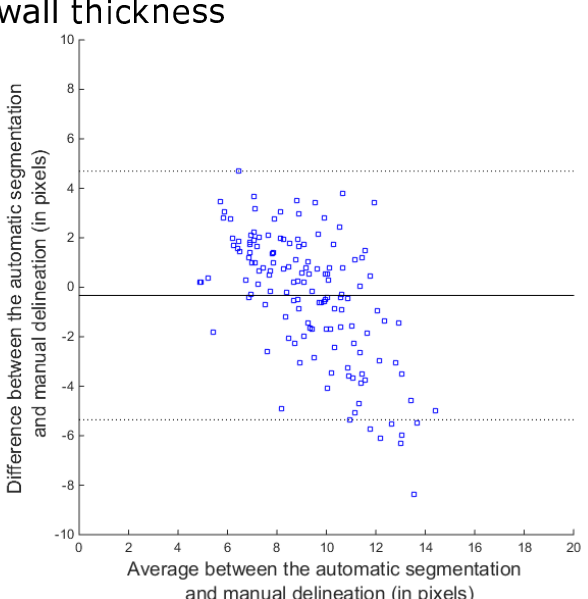

(b)

Figure 15: Linear regression (a) and Bland-Altman analysis (b) concerning the total carotid area, average carotid diameter and vessel wall thickness calculated from the carotid artery contours segmented by the Snake model with the NNGVF and the corresponding manual delineations. 

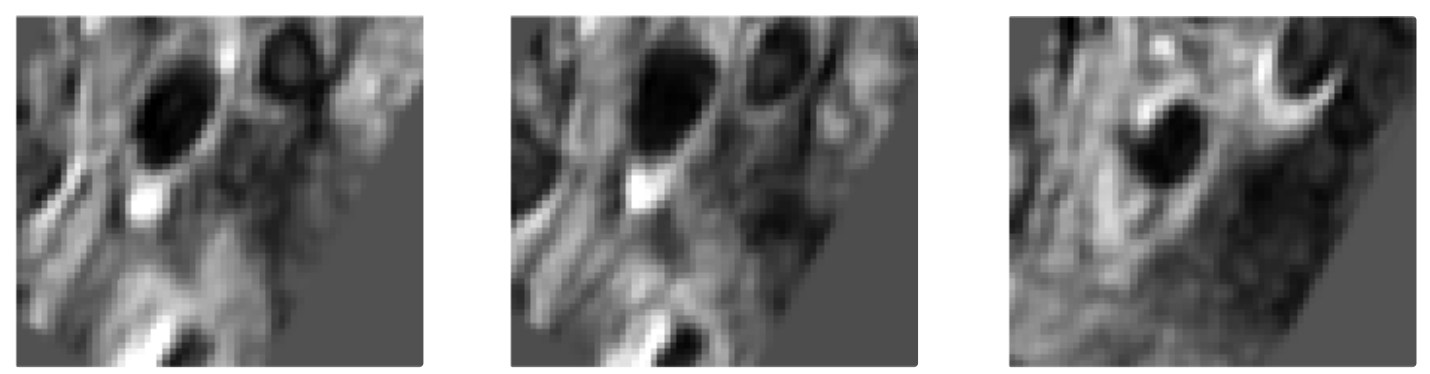

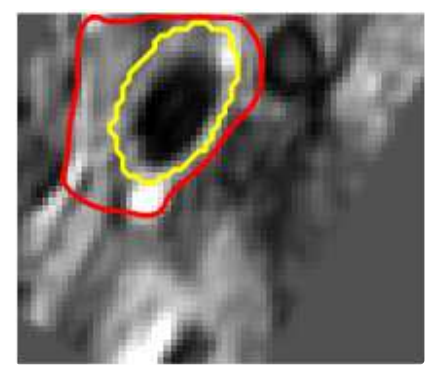

(a)

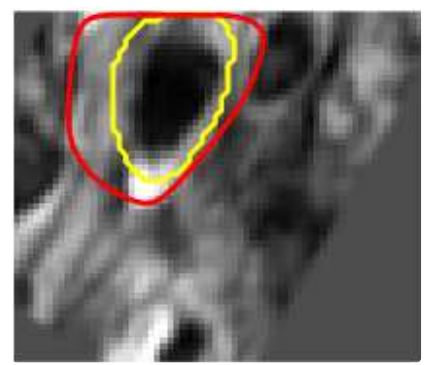

(b)

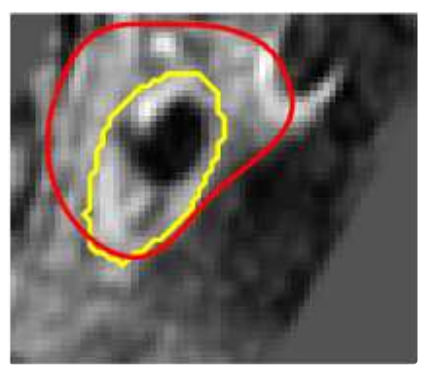

(c)

Figure 16: Examples of carotid artery boundaries segmented by the proposed approach in PDW MR images with almost indistinguishable boundaries: The images on the top row are the original PDW MR images and the ones on the bottom row shows the segmented carotid artery boundaries (in red) along with the corresponding manual delineations (in yellow). The differences found between the areas of the segmented and manually delineated carotid artery boundaries shown in images (a-c) were equal to 399 pixels, 297 pixels and 582 pixels, respectively. The images shown in (a) and (b) are indeed cropped as the majority ones provided with the dataset.

whether a significant difference exists between the results obtained from the proposed segmentation method and the ones of the manual delineations. Moreover, the calculated $p$-values are also important to assess the statistical analysis between the results of the proposed methods and the ones obtained from the NNGVF and the Weighted External Energy proposed by Khadidos et al. [40].

Table 6: Results of the Wilcoxon signed-rank test ( $p$-values) with $5 \%$ of significance for the Dice coefficient, Polyline Distance (PD), Mean Contour Distance (MCD), Centroid Distance (CD), Area and Average Diameter (AVD).

\begin{tabular}{lllllll}
\hline & Dice & PD & MCD & CD & Area & AVD \\
\hline Lumen Aut. vs Lumen Manual & N/A & N/A & N/A & N/A & 0.58900 & 0.4489 \\
PWED-Manual & N/A & N/A & N/A & N/A & $3.4472 \mathrm{e}-07$ & $9.9863 \mathrm{e}-07$ \\
NNGVF-Manual & N/A & N/A & N/A & N/A & $4.2461 \mathrm{e}-05$ & $1.8380 \mathrm{e}-05$ \\
WED-Manual & N/A & N/A & N/A & N/A & $4.2629 \mathrm{e}-15$ & $3.8329 \mathrm{e}-14$ \\
PWED-NNGVF & 0.0002 & 0.6652 & 0.0017 & 0.0005 & $4.7934 \mathrm{e}-28$ & $5.4811 \mathrm{e}-27$ \\
PWED-WED & $6.4248 \mathrm{e}-10$ & $2.6847 \mathrm{e}-09$ & $6.3462 \mathrm{e}-09$ & $1.0209 \mathrm{e}-06$ & $5.0742 \mathrm{e}-16$ & $3.8785 \mathrm{e}-16$ \\
\hline
\end{tabular}

*NNGVF = Neighbourhood-Extending and Noise-Smoothing Gradient Vector Flow; N/A = Not Applicable

$*$ PWED $=$ Proposed Weighted External Energy; WED = Weighted External Energy proposed by Khadidos et al. [40].

Regarding the lumen segmentation, the results presented in Table 6 indicate that the proposed automatic method produced similar results in comparison with the corresponding manual delineations in terms of area 
and average diameter of the segmented lumens. One can highlight that the results are quite similar at $5 \%$ of significance, which shows the similarities between the automatic and manual segmented lumens. On the other hand, the statistical analysis of the segmented carotid wall boundaries indicates a difference between the areas and average diameters calculated from both automatic and manual delineations. As previously discussed, such variances might be explained by the overestimation of the carotid wall boundaries produced by the proposed method in comparison with the ones manually delineated. Similarly, underestimation of such boundaries generated by the NNGVF external energy might also explain the discrepancies between its results and the corresponding manual delineations.

Despite such differences, the superior results produced by the method introduced here for carotid wall segmentation purpose are confirmed as shown by the low $p$-values calculated from the Wilcoxon signed-rank test (Table 6). One can notice the low $p$-values of all measures, particularly as to the Polyline Distance obtained from the proposed external energy and the NNGVF method, which indicates similarities between the distances calculated from both methods.

\subsection{Computational cost}

Table 7 presents the mean execution time of the Snake model performed with the ellipse constraint and each external energy over the set of images of each patient.

Table 7: Mean computational time (in seconds) of the Snake model considering the ellipse constraint and the proposed external energy in comparison with the NNGVF. The results are expressed as mean \pm standard deviation.

\begin{tabular}{lrrr}
\hline Patient & Ellipse constraint & NNGVF only & Proposed external energy \\
\hline Patient 1 & $4.16 \pm 0.58$ & $4.13 \pm 2.45$ & $3.93 \pm 2.39$ \\
Patient 2 & $2.33 \pm 0.90$ & $1.49 \pm 1.12$ & $4.12 \pm 1.73$ \\
Patient 3 & $2.06 \pm 1.07$ & $1.27 \pm 0.72$ & $2.25 \pm 1.02$ \\
Patient 4 & $2.75 \pm 3.97$ & $0.60 \pm 0.63$ & $4.50 \pm 5.11$ \\
Patient 5 & $2.07 \pm 0.77$ & $1.97 \pm 1.54$ & $4.71 \pm 2.99$ \\
Patient 6 & $1.76 \pm 0.94$ & $1.31 \pm 1.33$ & $6.91 \pm 5.05$ \\
Patient 7 & $2.07 \pm 0.49$ & $4.33 \pm 5.19$ & $3.44 \pm 1.66$ \\
Patient 8 & $2.63 \pm 0.88$ & $2.21 \pm 0.97$ & $3.97 \pm 3.66$ \\
Patient 9 & $2.04 \pm 1.33$ & $2.55 \pm 3.66$ & $6.15 \pm 3.06$ \\
Patient 10 & $2.00 \pm 0.86$ & $1.98 \pm 1.74$ & $4.35 \pm 3.94$ \\
Patient 12 & $2.71 \pm 1.08$ & $3.03 \pm 2.59$ & $3.63 \pm 2.74$ \\
\hline
\end{tabular}

From the data in Table 7, one can notice that the computational time of the proposed method is occasionally greater than the one of the original Snake algorithm with only the NNGVF external energy. Such behavior is explained by the fast and incomplete convergence of the Snake model when the wall of the carotid artery under analysis is thick and inhomogeneous causing thereby, an incorrect segmentation (images in the 
middle row of Figure 10). However, there were cases where the obtained segmentation results and the required computational times were similar for both methods, as happened, for example, for Patient 1 (Table 77.

Regarding the computational complexity, the proposed active contour model performs similarly as the

original Snake algorithm, being the execution time of former method somehow influenced by the latter one. The computational cost of the new method might be calculated taking into account the following three steps: i) application of the Snake model with the ellipse constraint; ii) calculation of the proposed external energy (Equation 11); and iii) application of the Snake model over the image related to the external energy. As demonstrated by Liang et al. [44], the computational complexity of the Snake model is for each iteration $O(n)$, where $n$ is the set of points of the contour to be deformed. Furthermore, the ellipse constraint is performed similarly, ending up therefore with a computational complexity of $O(n)$ for each point in $n$. Regarding Equation 11] the external energy calculated by the NNGVF method has a computational cost of $O(M \times N)$ in each iteration, where $M$ and $N$ represent the number of rows and columns of the image, respectively. This computational complexity can be also given as $O\left(N^{2}\right)$ when the image resolution is $N$ x $N$ pixels. Finally, the computational cost of the third step is also $O(n)$ since it represents the application of the Snake model over the contour to be evolved towards the carotid artery boundary. Since the aforementioned steps i) and iii) are performed in the same contour to be deformed along the whole segmentation process, the computational complexity of the Snake model may be given as $O(n)$ for all the $n$ points of the contour in each iteration of its evolving process.

In summary, the computational complexity of the proposed segmentation method, including all the steps to detect the carotid wall boundary, can be expressed as $O(i \mathrm{x} n)+O\left(I \mathrm{x} N^{2}\right)$, where $i$ is the number of iterations of the Snake model, $n$ is the number of points of the contour to be evolved, $N$ represents the size of the input image and $I$ is the number of iterations of the NNGVF method.

\section{Discussion}

The development of automatic segmentation methods devoted to medical images plays an important role in providing experts with auxiliary diagnosis tools for identifying various types of diseases. For example, the segmentation of the lumen and carotid artery boundaries in MR images represents an important step to quickly identify and quantify potential atherosclerosis in arteries. The segmentation of carotid artery boundaries in PDW MR images was successfully tackled in this study. The use of the images obtained by the gray-weighted distance to expand the lumen contour beyond the boundary of the lumen region in the input image represents an important contribution to correctly identify the boundary of the carotid artery. Additionally, the usage of the proposed weighted external energy in the Snake model proved to be effective in expanding the lumen contours towards the boundaries of carotid arteries even in cases of large thickness. Additionally, the modification in the external energy as proposed by Khadidos et al. [40] makes the convergence of the lumen contours more robust, stable and appropriate for carotid arteries with weak 
image edges.

At first, the proposed segmentation method is adaptable to other image modalities without significant changes in the values of its parameters. However, this study aimed at focusing primarily on 2D MR images, namely on the ones from a cross-sectional perspective, since the boundaries of the carotid arteries acquired by this image modality are usually readily distinguishable, especially the carotid wall boundary. A substantial number of studies, with particular reference to the ones presented by Cheng et al. [45] and Luo et al. [46], just to cite a few, have also been carried out considering the usage of specific image modalities to identify vessel structures either in longitudinal or cross-sectional views. However, this study was specifically conducted to address the segmentation of the carotid artery in cross-sectional views, without including other images modalities or prior steps to handle 3D imaging volumes as presented by Cheng et al. 45], which justifies the dataset herein used.

\subsection{Parameters initialization}

The convergence of the lumen contour to the boundary of the carotid artery under analysis depends on the value of parameter $\alpha$ defined for the elasticity term of the Snake model. When the value of $\alpha$ decreases, the contour elasticity is increased, leading to an easier evolution towards the boundary of interest. On the other hand, the contour becomes more rigid as the value of $\alpha$ increases, leading the contour to deflate even in regions with large capture range provided by the gradient vectors. Hence, it was decided to use a low value for parameter $\alpha$ in order to avoid the swift convergence and shrinking of the lumen contour. Parameter $\beta$ is employed to control the smoothness and bend of the contour. The higher is the value of $\beta$, the more smooth the contour is. In the second step of the proposed approach, the value of $\beta$ was set to 1 (one) in order to smooth the final contour of the carotid artery boundary. Parameter $\mu$ represents a regularization term that controls the tradeoff between the Laplacian operator and the gradient of the image edge map defined for the NNGVF. The value of $\mu$ depends on the amount of noise in the image under analysis. The higher is the value of $\mu$, more diffuse are the gradient vectors. In this study, the low value of $\mu$ determined by the parameter tuning procedure plays an important role to avoid the excessive diffusion of the gradient vectors and the evolution of the lumen contour beyond the boundary of the carotid artery under analysis. Parameter $\kappa$ is responsible for the strength of the balloon forces and therefore, used to inflate or deflate the contour to be deformed. When the value of $\kappa$ increases, the amplitude of the unit vectors defined by the balloon force also increases, leading the contour to pass through weak edges. Since the lumen contour needs to be inflated from the lumen region to the boundary of the carotid artery, the value of $\kappa$ was set 1 (one) to avoid the excessive amplitude of the balloon forces. Parameter $\sigma$ is used in the Gaussian filter and has an important role to control the amount of smoothness of the image and the capture range of the gradient vectors. A large value for $\sigma$ will cause the boundaries to become more smooth and distorted and therefore, the capture range of the gradient vectors will be also increased. In order to avoid excessive distortion of the boundaries of the input image, the value of $\sigma$ was set to 1 (one). The number of iterations defined for the first step of the carotid artery boundary segmentation was lower than the one of the second step due to the computational 
cost imposed by the ellipse constraint. However, the value chosen in this study was sufficient to expand successfully the lumen contour beyond the boundary of the lumen region. The radius of the disk-shaped template used to calculate the values of $A D$ and $h$ defined in Equations 7 and 8 plays an important role in the correct determination of the carotid artery boundary. Since the entropy of the pixels ahead the lumen contour to be expanded is responsible for the convergence to the carotid artery boundary of interest, a large number of pixels could increase the entropy and lead to a fast and incomplete evolution of the lumen contour. Contrary, a low number of pixels may still decrease the entropy defined in Equation 9 and, consequently, lead the lumen contour to leak the true boundary of the carotid artery. Hence, the parameter tuning procedure was important to determine the best radius for the dataset used in the experiments. The same values for $\alpha$, $\beta, \mu$ and $\sigma$ defined for the proposed weighted external energy were also used in the NNGVF formulation.

Although the values established for the parameters of the proposed approach were able to efficiently identify the boundaries of the carotid arteries in the images used, the convergence of the lumen contour is mostly dependent on the parameters of the internal energy of the Snake model, the amplitude of the balloon forces and the number of iterations used to perform the convergence of the contour until it fits the desirable boundary. Changing the image resolution may still affect the segmentation results, leading the lumen contour to an incomplete and slow convergence in images of higher resolutions. However, the proposed approach might be easily adapted to initialize and adjust the aforementioned parameters for other MR image datasets.

\subsection{Performance of the proposed approach}

The distance map proposed in this study proved to be effective in expanding the lumen contour beyond the boundary of the lumen region and therefore, to avoid the gradient vectors that can hinder the contour to converge to the boundary of the carotid artery under analysis. Unlike the grayscale intensity of the MR image, which contains variations in the gradient vectors obtained by external forces, the gray-weighted distance map provides a gradual increase of the grayscale intensities starting from the centre of the lumen to the borders of the assessed image. Hence, it provides undisturbed and low grayscale intensities near the lumen region of the carotid artery, which helps the lumen contour to expand outside the lumen region owing to the low magnitude of the gradient vectors in that region. Along with the ellipse constraint, the contour resultant from the Snake model applied to the image representing the distance map becomes more regular and without concavities that may be caused by low intensity regions close to the lumen of the carotid artery.

The modification in the weighted external energy as suggested by Khadidos et al. 40] shown to be successful in converging the lumen contours to the boundaries of carotid arteries with large thickness. The evolution of the lumen contour may still fail due to the diffusion of the gradient vectors in regions with large intensity variations. Balloon forces have been proposed to improve the convergence of the contour based on the strength of the unit vectors in each pixel of the contour to be expanded, leading to a rapid convergence even in regions with large areas. However, the balloon forces still fail in fitting the contour in regions having weak edges, which causes the contour to leak the boundary of interest. Hence, the combination of balloon 
forces and the GVF-based external energy represents an important approach to handle at the same time with the quick evolution of the contour in regions with large areas and the decrease of the contour evolution in weak 66 improves the convergence of the lumen contour in weak edges of the carotid artery boundary of interest due to the sensitivity of the entropy in regions with high variations of grayscale intensities. Additionally, the penalty term defined in Equation 10 proved to be effective in decreasing the contour evolution when dark regions corresponding to the background of the image are found.

the Dice coefficient between the carotid artery boundaries obtained by our approach and the corresponding contours manually delineated was equal to $0.83 \pm 0.11(82.61 \% \pm 10.84 \%)$. The Jaccard similarity obtained from the comparison between the carotid arteries segmented by the proposed approach and the corresponding 
manual delineations was equal to $0.72 \pm 0.15$. The main limitation of the study carried out by van 't Klooster

et al. 15] is that a signal profile vector with a specific length is necessary to find the edges of the lumen and carotid artery boundaries. Additionally, the gradient information is insufficient to cope with weak edges commonly found in MR images of carotid arteries. Although the approach proposed by Saba et al. [17] is similar to the one proposed in this study, it has a main limitation: the expansion of the lumen contour beyond the boundary of the lumen region is based on a heuristic value. Similarly, the method proposed by Gao et al. 18] also relies on the expansion of the lumen contour based on a heuristic value, although the method is fully automatic. Contrary, our proposed approach is fully automatic and the expansion of the lumen contours is completely performed by using the distance map without needing any heuristic value.

\subsection{Limitations}

The proposed approach has two limitations. The first one regards the segmentation of carotid arteries with calcifications. Calcifications appears as dark regions in PDW MR images and therefore, the convergence of the lumen contours towards the boundaries of carotid arteries may be compromised due to the strength of the gradient vectors that avoid the expansion of the contour when calcified regions are presented. Hence, future studies will be conducted to effectively identify local regions inside the carotid artery associated to calcifications. The second limitation regards the segmentation of the lumen and carotid artery boundaries in regions with bifurcations. Although bifurcation regions have been identified and delineated by the physician in the PDW MR images, the carotid artery boundary manually delineated in the input image having the higher Dice coefficient value with the segmented carotid artery boundary was chosen to evaluate the accuracy of the result. Hence, the segmentation of the lumen and carotid artery boundaries in bifurcation regions is expected to be considered in future researches. Although Deep Learning approaches have been a topic of research highly discussed and used in many studies related to image segmentation and classification tasks (47, 48, 49, 50, 51, 52, 53]), the proposed method presented here is aimed for the identification of structures in medical images by means of well-known image processing and analysis algorithms. However, we also intend to consider Deep Learning techniques for the segmentation of the structures of the carotid arteries in future works.

\section{Conclusions}

The segmentation of the lumen and carotid artery boundaries plays an important role in assessing the progression of atherosclerosis. An automatic approach for the segmentation of carotid artery boundary in PDW MR images was proposed in this article. The main contribution of this study is an efficient and robust approach based on the gray-weighted distance map to expand the lumen contour towards the boundary of the carotid artery without the use of any heuristic values. Additionally, the lumen contour expanded by the Snake model with the ellipse constraint applied to the distance map image, is used to identify the final boundary of the carotid artery based on the Snake model along with the modified weighted external energy. 
The proposed approach proved to be also robust in identifying the boundaries of carotid arteries that have large thickness and weak image edges.

The comparison between the carotid artery boundaries segmented by the proposed approach and the corresponding manual delineations showed that the usage of the proposed weighted external energy in the Snake model is more effective than if only the NNGVF is used. Additionally, the proposed approach outperformed the results reported in related studies found in the literature.

Futures studies will be conducted to efficiently segment the lumen and carotid artery boundaries in PDW MR images having bifurcation regions. The segmentation of the carotid artery boundaries using other MR image datasets is also expected in order to assess the parameters tuning procedure and the segmentation results in images obtained with higher resolutions and different acquisition settings. Moreover, the segmentation of the boundaries of carotid arteries having calcified regions is also expected to be addressed in order to avoid the local convergence and incomplete evolution of the segmented lumen contours that can occur in these cases.

\section{Acknowledgements}

This work was partially funded by "Coordenação de Aperfeiçoamento de Pessoal de Nível Superior" (CAPES), funding agency in Brazil, under the PhD Grant with reference number 0543/13-6.

The authors thank the funding of Project NORTE-01-0145-FEDER-000022 - SciTech - Science and Technology for Competitive and Sustainable Industries, co-financed by "Programa Operacional Regional do Norte" (NORTE2020), through "Fundo Europeu de Desenvolvimento Regional" (FEDER).

\section{References}

[1] S. Mendis, P. Puska, B. Norrving, Global atlas on cardiovascular disease prevention and control, World Health Organization, 2011.

[2] D. S. Jodas, A. S. Pereira, J. M. R. S. Tavares, A review of computational methods applied for identification and quantification of atherosclerotic plaques in images, Expert Systems with Applications 46 (2016) 1-14.

[3] L. Esposito, M. Sievers, D. Sander, P. Heider, O. Wolf, O. Greil, C. Zimmer, H. Poppert, Detection of unstable carotid artery stenosis using MRI, Journal of Neurology 254 (2007) 1714-1722.

[4] U. R. Acharya, M. R. K. Mookiah, S. Vinitha Sree, D. Afonso, J. Sanches, S. Shafique, A. Nicolaides, L. M. Pedro, J. Fernandes e Fernandes, J. S. Suri, Atherosclerotic plaque tissue characterization in 2D ultrasound longitudinal carotid scans for automated classification: a paradigm for stroke risk assessment, Medical \& Biological Engineering \& Computing 51 (2013) 513-523. 
[5] L. Esposito-Bauer, T. Saam, I. Ghodrati, J. Pelisek, P. Heider, M. Bauer, P. Wolf, A. Bockelbrink, R. Feurer, D. Sepp, C. Winkler, P. Zepper, T. Boeckh-Behrens, M. Riemenschneider, B. Hemmer, H. Poppert, MRI plaque imaging detects carotid plaques with a high risk for future cerebrovascular events in asymptomatic patients, PLoS ONE 8 (2013) e67927.

[6] A. Arias, J. Petersen, A. van Engelen, H. Tang, M. Selwaness, J. C. M. Witteman, A. van der Lugt, W. Niessen, M. de Bruijne, Carotid Artery Wall Segmentation by Coupled Surface Graph Cuts, Springer Berlin Heidelberg, Berlin, Heidelberg, 2013, pp. 38-47.

[7] A. M. Arias-Lorza, J. Petersen, A. van Engelen, M. Selwaness, A. van der Lugt, W. J. Niessen, M. de Bruijne, Carotid artery wall segmentation in multispectral MRI by coupled optimal surface graph cuts, IEEE Transactions on Medical Imaging 35 (2016) 901-911.

[8] I. M. Adame, R. J. van der Geest, B. A. Wasserman, M. Mohamed, J. H. Reiber, B. P. Lelieveldt, Automatic plaque characterization and vessel wall segmentation in magnetic resonance images of atherosclerotic carotid arteries, in: Medical Imaging 2004, International Society for Optics and Photonics, 2004, pp. $265-273$.

[9] I. M. Adame, R. J. van der Geest, B. A. Wasserman, M. A. Mohamed, J. H. Reiber, B. P. Lelieveldt, Automatic segmentation and plaque characterization in atherosclerotic carotid artery MR images, Magnetic Resonance Materials in Physics, Biology and Medicine 16 (2004) 227-234.

[10] Fang Wang, Qiu Guan, S. Chen, Zhongzhao Teng, U. Sadat, J. Gillard, Multi-scale segmentation of carotid artery wall in MRI images, in: The 2nd International Conference on Information Science and Engineering, IEEE, 2010, pp. 1-4.

[11] C. Yuan, E. Lin, J. Millard, J. N. Hwang, Closed contour edge detection of blood vessel lumen and outer wall boundaries in black-blood MR images, Magnetic Resonance Imaging 17 (1999) 257-266.

[12] H. M. Ladak, J. B. Thomas, J. R. Mitchell, B. K. Rutt, D. A. Steinman, A semi-automatic technique for measurement of arterial wall from black blood MRI, Medical Physics 28 (2001) 1098-1107.

[13] G. Adams, G. W. Vick III, C. Bordelon, W. Insull, J. Morrisett, An algorithm for quantifying advanced carotid artery atherosclerosis in humans using MRI and active contours, in: Proc. SPIE, volume 4684, International Society for Optics and Photonics, 2002, pp. 1448-1457.

[14] H. Tang, R. S. van Onkelen, T. van Walsum, R. Hameeteman, M. Schaap, F. L. Tori, Q. J. A. van den Bouwhuijsen, J. C. M. Witteman, A. van der Lugt, L. J. van Vliet, W. J. Niessen, A Semi-automatic Method for Segmentation of the Carotid Bifurcation and Bifurcation Angle Quantification on Black Blood MRA, Springer Berlin Heidelberg, Berlin, Heidelberg, 2010, pp. 97-104. 
[15] R. van 't Klooster, P. J. de Koning, R. A. Dehnavi, J. T. Tamsma, A. de Roos, J. H. Reiber, R. J. van der Geest, Automatic lumen and outer wall segmentation of the carotid artery using deformable three-dimensional models in MR angiography and vessel wall images, Journal of Magnetic Resonance Imaging 35 (2012) 156-165.

[16] K. Hameeteman, R. van 't Klooster, M. Selwaness, A. van der Lugt, J. C. M. Witteman, W. J. Niessen, S. Klein, Carotid wall volume quantification from magnetic resonance images using deformable model fitting and learning-based correction of systematic errors, Physics in Medicine and Biology 58 (2013) $1605-1623$.

[17] L. Saba, H. Gao, E. Raz, S. V. Sree, L. Mannelli, N. Tallapally, F. Molinari, P. P. Bassareo, U. R. Acharya, H. Poppert, J. S. Suri, Semiautomated analysis of carotid artery wall thickness in MRI, Journal of Magnetic Resonance Imaging 39 (2014) 1457-1467.

[18] S. Gao, R. van 't Klooster, A. Brandts, S. D. Roes, R. Alizadeh Dehnavi, A. de Roos, J. J. Westenberg, R. J. van der Geest, Quantification of common carotid artery and descending aorta vessel wall thickness from MR vessel wall imaging using a fully automated processing pipeline, Journal of Magnetic Resonance Imaging 45 (2017) 215-228.

[19] S. Zhao, Z. Gao, H. Zhang, Y. Xie, J. Luo, D. Ghista, Z. Wei, X. Bi, H. Xiong, C. Xu, S. Li, Robust segmentation of intima-media borders with different morphologies and dynamics during the cardiac cycle, IEEE Journal of Biomedical and Health Informatics 22 (2018) 1571-1582.

[20] P. P. R. Filho, A. C. da Silva Barros, J. S. Almeida, J. Rodrigues, V. H. C. de Albuquerque, A new effective and powerful medical image segmentation algorithm based on optimum path snakes, Applied Soft Computing 76 (2019) $649-670$.

[21] J. Cheng, Y. Chen, Y. Yu, B. Chiu, Carotid plaque segmentation from three-dimensional ultrasound images by direct three-dimensional sparse field level-set optimization, Computers in Biology and Medicine $94(2018) 27-40$.

[22] V. N. Naik, R. Gamad, P. Bansod, Efficient initialisation of distance-regularised level set without reinitialisation scheme and quantitative evaluation of IMT in B mode ultrasound common carotid artery images, Computer Methods in Biomechanics and Biomedical Engineering: Imaging \& Visualization 7 (2019) 207-226.

[23] Y. Yang, Z. Zhuang, A. N. Josephraj, An improved ultrasound image segmentation method based on level set, in: X. Jiang, J.-N. Hwang (Eds.), Tenth International Conference on Digital Image Processing (ICDIP 2018), volume 10806, International Society for Optics and Photonics, SPIE, 2018, pp. 1496 1500. 
[24] J. Wang, Y. Cheng, C. Guo, Y. Wang, S. Tamura, Shape-intensity prior level set combining probabilistic atlas and probability map constrains for automatic liver segmentation from abdominal CT images, International Journal of Computer Assisted Radiology and Surgery 11 (2016) 817-826.

[25] D. S. Jodas, A. S. Pereira, J. M. R. S. Tavares, Automatic segmentation of the lumen region in intravascular images of the coronary artery, Medical Image Analysis 40 (2017) 60-79.

[26] A. van Engelen, W. J. Niessen, S. Klein, H. C. Groen, H. J. M. Verhagen, J. J. Wentzel, A. van der Lugt, M. de Bruijne, Atherosclerotic plaque component segmentation in combined carotid MRI and CTA data incorporating class label uncertainty, PLoS ONE 9 (2014) 1-14.

[27] K. Qian, S. Cao, P. Bhattacharya, Skeletonization of gray-scale images by gray weighted distance transform, in: AeroSense'97, International Society for Optics and Photonics, 1997, pp. 224-228.

[28] D. Baggett, M.-a. Nakaya, M. McAuliffe, T. P. Yamaguchi, S. Lockett, Whole cell segmentation in solid tissue sections, Cytometry Part A 67 (2005) 137-143.

[29] R. Strand, Minimal paths by sum of distance transforms, in: International Conference on Discrete Geometry for Computer Imagery, Springer, 2016, pp. 349-358.

[30] P. Soille, Generalized geodesy via geodesic time, Pattern Recognition Letters 15 (1994) 1235-1240.

[31] T. McInerney, D. Terzopoulos, Deformable models in medical image analysis: a survey, Medical Image Analysis 1 (1996) 91-108.

[32] Z. Ma, J. M. R. S. Tavares, R. N. Jorge, T. Mascarenhas, A review of algorithms for medical image segmentation and their applications to the female pelvic cavity, Computer Methods in Biomechanics and Biomedical Engineering 13 (2010) 235-246.

[33] H. Tang, T. van Walsum, R. Hameeteman, M. Schaap, A. van der Lugt, L. J. van Vliet, W. J. Niessen, Lumen segmentation of atherosclerotic carotid arteries in CTA, in: 9th IEEE International Symposium on Biomedical Imaging (ISBI), IEEE, 2012, pp. 274-277.

[34] A. M. F. Santos, R. M. dos Santos, P. M. A. Castro, E. Azevedo, L. Sousa, J. M. R. S. Tavares, A novel automatic algorithm for the segmentation of the lumen of the carotid artery in ultrasound B-mode images, Expert Systems with Applications 40 (2013) 6570-6579.

[35] A. M. F. Santos, J. M. R. S. Tavares, L. Sousa, R. Santos, P. Castro, E. Azevedo, Automatic segmentation of the lumen of the carotid artery in ultrasound B-mode images, Proc. SPIE 8670 (2013) 86703I-186703I-16.

[36] M. Kass, A. Witkin, D. Terzopoulos, Snakes: Active contour models, International Journal of Computer Vision 1 (1988) 321-331. 
[37] L. D. Cohen, On active contour models and balloons, CVGIP: Image Understanding 53 (1991) 211 218.

[48] Y. Dai, Z. Tang, Y. Wang, Z. Xu, Data Driven Intelligent Diagnostics for Parkinson's Disease, IEEE Access (2019) 1-1.

[49] S. Sudha, K. B. Jayanthi, C. Rajasekaran, N. Madian, T. Sunder, Convolutional Neural Network for Segmentation and Measurement of Intima Media Thickness, Journal of Medical Systems 42 (2018).

[38] C. Xu, J. Prince, Snakes, shapes, and gradient vector flow, IEEE Transactions on Image Processing 7 (1998) 359-369.

[39] L. Liu, A. C. Bovik, Active contours with neighborhood-extending and noise-smoothing gradient vector flow external force, EURASIP Journal on Image and Video Processing 2012 (2012) 1-6.

[40] A. Khadidos, V. Sanchez, C.-T. Li, Active contours based on weighted gradient vector flow and balloon forces for medical image segmentation, in: 2014 IEEE International Conference on Image Processing (ICIP), IEEE, 2014, pp. 902-906.

[41] N. Ray, S. T. Acton, K. Ley, Tracking leukocytes in vivo with shape and size constrained active contours, IEEE Transactions on Medical Imaging 21 (2002) 1222-1235.

[42] Y. Wang, Y. Wu, Y. Jia, Shape constraints for the left ventricle segmentation from cardiac cine MRI based on snake models, in: Shape Analysis in Medical Image Analysis, Springer, 2014, pp. 373-412.

[43] R. Kimmel, Numerical Geometry of Images: Theory, Algorithms, and Applications, SpringerVerlag, 2003.

[44] J. Liang, T. McInerney, D. Terzopoulos, United Snakes, Medical Image Analysis 10 (2006) 215-233.

[45] Y. Cheng, X. Hu, J. Wang, Y. Wang, S. Tamura, Accurate vessel segmentation with constrained b-snake, IEEE Transactions on Image Processing 24 (2015) 2440-2455.

[46] L. Luo, S. Liu, X. Tong, P. Jiang, C. Yuan, X. Zhao, F. Shang, Carotid artery segmentation using level set method with double adaptive threshold (DATLS) on TOF-MRA images, Magnetic Resonance Imaging 63 (2019) 123-130.

[47] F. Arcadu, F. Benmansour, A. Maunz, J. Willis, Z. Haskova, M. Prunotto, Deep learning algorithm predicts diabetic retinopathy progression in individual patients, npj Digital Medicine (2019).

] Y. Wang, Y. Qiu, T. Thai, K. Moore, H. Liu, B. Zheng, A two-step convolutional neural network based computer-aided detection scheme for automatically segmenting adipose tissue volume depicting on CT images, Computer Methods and Programs in Biomedicine 144 (2017) 97-104. 
[51] M. Xu, S. Qi, Y. Yue, Y. Teng, L. Xu, Y. Yao, W. Qian, Segmentation of lung parenchyma in CT images using CNN trained with the clustering algorithm generated dataset 08 Information and Computing Sciences 0801 Artificial Intelligence and Image Processing Robert Koprowski, BioMedical Engineering Online 18 (2019).

[52] X. Zhao, S. Qi, B. Zhang, H. Ma, W. Qian, Y. Yao, J. Sun, Deep CNN models for pulmonary nodule classification: model modification, model integration, and transfer learning, Journal of X-Ray Science and Technology (2019) 1-15.

825

[53] R. Zhou, A. Fenster, Y. Xia, J. D. Spence, M. Ding, Deep learning-based carotid media-adventitia and lumen-intima boundary segmentation from three-dimensional ultrasound images, Medical Physics 46 (2019) 3180-3193. 